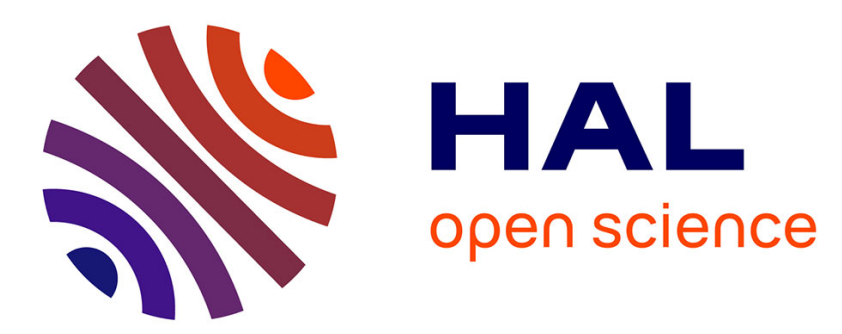

\title{
Noise and vibration for a self-excited mechanical system with friction
}

\author{
Kevin Soobbarayen, Sébastien Besset, Jean-Jacques Sinou
}

\section{To cite this version:}

Kevin Soobbarayen, Sébastien Besset, Jean-Jacques Sinou. Noise and vibration for a selfexcited mechanical system with friction. Applied Acoustics, 2013, 74 (10), pp.1191-1204. 10.1016/j.apacoust.2013.03.008 . hal-00826162

\section{HAL Id: hal-00826162 \\ https://hal.science/hal-00826162}

Submitted on 27 May 2013

HAL is a multi-disciplinary open access archive for the deposit and dissemination of scientific research documents, whether they are published or not. The documents may come from teaching and research institutions in France or abroad, or from public or private research centers.
L'archive ouverte pluridisciplinaire HAL, est destinée au dépôt et à la diffusion de documents scientifiques de niveau recherche, publiés ou non, émanant des établissements d'enseignement et de recherche français ou étrangers, des laboratoires publics ou privés. 


\title{
Noise and vibration for a self-excited mechanical system with friction
}

\author{
K. Soobbarayen ${ }^{1, a}$, S. Besset ${ }^{1, b}$, and J-.J. Sinou ${ }^{1, c}$ \\ ${ }^{1}$ Laboratoire de Tribologie et Dynamique des Systèmes, UMR CNRS 5513, \\ Ecole Centrale de Lyon, 36 avenue Guy de Collongue 69134 Ecully Cedex, France \\ $\left\{{ }^{\mathrm{a}}\right.$ kevin.soobbarayen, ${ }^{\mathrm{b}}$ sebastien.besset, ${ }^{\mathrm{c}}$ jean-jacques.sinou\}@ec-lyon.fr
}

\begin{abstract}
This work proposes a complete characterization of brake squeal from the calculation of the non-linear vibration to the calculation of the associated sound pressure. A simplified finite elements brake system model composed of a disc and a pad is investigated. The contact is modelled by introducing several local contact elements at the friction interface and a cubic contact law is used to describe the contact force. The classical Coulomb law is applied to model friction and the friction coefficient is assumed to be constant. The stability analysis of this system provides two classical cases of instabilities which are single and multiinstabilities. For one and two unstable modes, non-linear time integrations and spectrum analysis are performed to detect all the harmonic components in the velocity spectrum. In this paper, the decomposition by harmonic components of the velocity is used to calculate the acoustic radiation by applying the boundary element method for each contributions. The sound pressure radiated is calculated for the two cases under study and a comparison in terms of levels and directivity is provided. It can be noted that the two unstable modes case presents significantly higher levels of acoustic pressure. In near field, directivity patterns for both cases are composed of four main lobes with different orientations. Moreover, over others observation plan, the multi-instabilities case presents a more complex directivity pattern due to the participation of two modes in the time response. Finally, the study of the influence of the truncation shows that for both cases, the first two harmonic components are enough to describe to global pressure field with a good accuracy.
\end{abstract}

\section{Introduction}

Brake squeal phenomenon is one of the most complex and important problem in industry (see $[1,2])$. Actually, squeal events generate noise emissions that industrials need to eliminate or, at least, reduce in order to improve acoustic convenience. The mechanisms generating squeal are very complex and involve both the micro-structure of the friction interface and the macrostructure which interests many researchers as well. Several kinds of instabilities have been identified and many models have been proposed to reproduce this phenomenon as explained in $[3-7]$.

Researchers agree to say that squeal finds its origin at a friction interface between disc and pad that induces self-excited vibrations. Actually, non-linearities involving contact and friction can generate modes coalescence that could lead to instability. Several works have been performed to identify all the parameters which influence squeal. As showed in $[8,9]$, structural damping directly impacts squeal occurrences. Hydraulic pressure associated to braking process and contact friction parameters are also important. Even after all these works, the brake squeal phenomenon remains an issue which is very sensitive to many parameters making it a real challenge to characterize. 
However, numerical approaches can provide some results to predict squeal. Actually, the first step in this kind of study is to evaluate the stability of the system with respect to the friction coefficient. It consists in performing a complex eigenvalues analysis on the linearized system and detecting modes coalescence. Then, the Lyapunov theorem gives the stability of the non-linear system near a given sliding equilibrium configuration. However, this kind of analysis is not enough to be predictive [10]: the stability analysis may lead to an underestimation or an over-estimation of the unstable modes observed in the non-linear time simulation. Therefore, a numerical resolution of the complete non-linear system has to be performed in addition to the stability analysis to estimate the non-linear behaviour of the solution far from the sliding equilibrium [11-13]. With time responses, some features of squeal can be characterized, such as amplitudes of velocity or frequencies which composed the response. Although there have been some progresses, it is still impossible to eliminate squeal.

Experimental works can also provide a characterization of squeal as explained in [14-16]. Actually, it is possible to reproduce squeal events in laboratory and to determine the amplitudes of vibrations and the frequency spectrum. Moreover, the sound pressure levels can be determined during these experiments but it is still difficult to evaluate the influence of parameters on these levels. Besides, only few studies propose to numerically estimate the sound pressure levels.

In this paper, a method is proposed which aims at numerically evaluating sound pressure levels generated during brake squeal phenomenon. Section 2 presents the finite element model of a simplified brake system which is able to reproduce instabilities. Then, section 3 defines the classical tools of stability analysis which provide stable and unstable areas as a function of friction coefficient for the brake system under study. Section 4 presents the developed method which calculates the sound pressure field. Finally, section 5 presents time history responses and the propagation of the sound pressure wave.

\section{Brake system model}

\subsection{Finite element model}

In this paper, a simplified brake system model is under study. It is composed of the two main components which contribute to the brake squeal phenomenon: a disc and a pad (see Fig. 1). There are about 12000 nodes and height-node linear hexahedron elements are used. The outline of the upper surface of the pad can only translates along the normal direction. The inner surface of the disc is clamped. Moreover, a hydraulic pressure is applied to the back-plate of the pad.

Over the interface between the disc and the pad, nine uniformly spaced contact points (see Fig. 1b) are selected. The limited number of contact points has been chosen for the sake of simplicity (following the previous paper of Coudeyras et al. [17]) due to the fact that the main objective of the paper is to propose a complete framework for brake squeal including not only the calculation of the non-linear self-excited vibrations but also the associated brake sound pressure. In this context, we assume that the simplified brake model is sufficient to illustrate our methodology (i.e. analysis of friction-induced vibration from non-linear vibrations to noise). The limited number of contact points may be a reduction of the uniformly distributed contact law over the contact area. So, the friction interface is modelled by introducing contact elements on the previously selected points. Contact and loss of contact configurations at the friction interface are taken into account so that the disc and the pad can separate at several local nodes during vibrations. Moreover, the contact force is described with a cubic law in order to approximate the first and third order of the classical pad compression curves as explained in [10]. The non-linear 


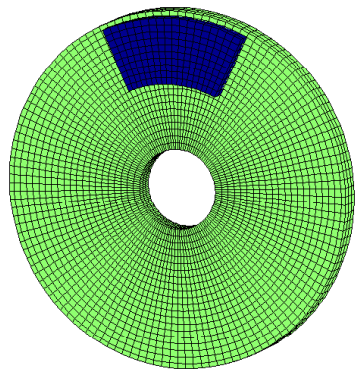

(A)

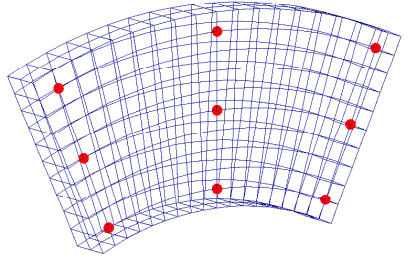

(в)

Figure 1: Finite element model of the brake system. (a) simplified brake system; (b) pad and contact nodes

contact force vectors at the friction interface along the normal $z$-direction are defined by

$$
\begin{gathered}
F_{\text {contact }, z}^{d}=\left\{\begin{array}{l}
k_{L}\left(X_{d}-X_{p}\right)+k_{N L}\left(X_{d}-X_{p}\right)^{3} \quad \text { if }\left(X_{p}-X_{d}\right)>0 \\
0 \text { otherwise }
\end{array}\right. \\
F_{\text {contact }, z}^{p}=-F_{\text {contact }, z}^{d}
\end{gathered}
$$

where $d$ and $p$ define the disc and the pad respectively, $k_{L}$ and $k_{N L}$ correspond to the linear and non-linear contact stiffnesses at the friction interface between the disc and the pad, and $X_{d}$ and $X_{p}$ are the displacements along the normal direction of the friction interface. The friction coefficient $\mu$ is assumed to be constant for the sake of simplicity and the classical Coulomb law is applied. So the non-linear friction force vectors at the friction interface in the tangential plan are defined by

$$
\begin{gathered}
\left\{\begin{array}{l}
F_{\text {friction }, x}^{d}=\mu F_{\text {contact }, z}^{d} \operatorname{sign}\left(v_{r}\right) \mathbf{e}_{\theta} \cdot \mathbf{x} \\
F_{\text {friction }, y}^{d}=\mu F_{\text {contact }, z}^{d} \operatorname{sign}\left(v_{r}\right) \mathbf{e}_{\theta} \cdot \mathbf{y}
\end{array}\right. \\
\left\{\begin{array}{l}
F_{\text {friction }, x}^{p}=-F_{\text {friction }, x}^{d} \\
F_{\text {friction }, y}^{p}=-F_{\text {friction }, y}^{d}
\end{array}\right.
\end{gathered}
$$

where $v_{r}$ is the relative velocity between the disc and the pad, $\mathbf{x}$ and $\mathbf{y}$ are the tangential directions of the friction interface, $\mathbf{e}_{\theta}$ is the orthoradial direction of the disc (Fig. 5). During the braking process, it is assumed that the pad is always sliding over the disc. The velocity $v_{r}$ corresponds to the relative velocity between the disc and the pad along $\mathbf{e}_{\theta}$ direction (i.e. including the vibration motion of the system) and also depends on the contact points.

Finally, the brake system is reduced by using a Craig and Bampton method. All the dofs associated to the selected contact nodes at the friction interface are retained, and the first hundred eigenmodes of the global brake system are conserved. The MAC criterion presented in Fig. 2a shows the correlation between the eigenvectors of the reduced and physical models. Fig. $2 \mathrm{~b}$ represents the relative error between the eigenfrequencies of the physical and reduced model. This reduction gives a very good correlation between the complete and the reduced models until $20 \mathrm{kHz}$.

Finally, the equations of motion for the reduced brake system are given by

$$
\mathbf{M} \ddot{\mathbf{X}}+\mathbf{C} \dot{\mathbf{X}}+\mathbf{K X}=\mathbf{F}_{\mathrm{NL}}+\mathbf{F}
$$




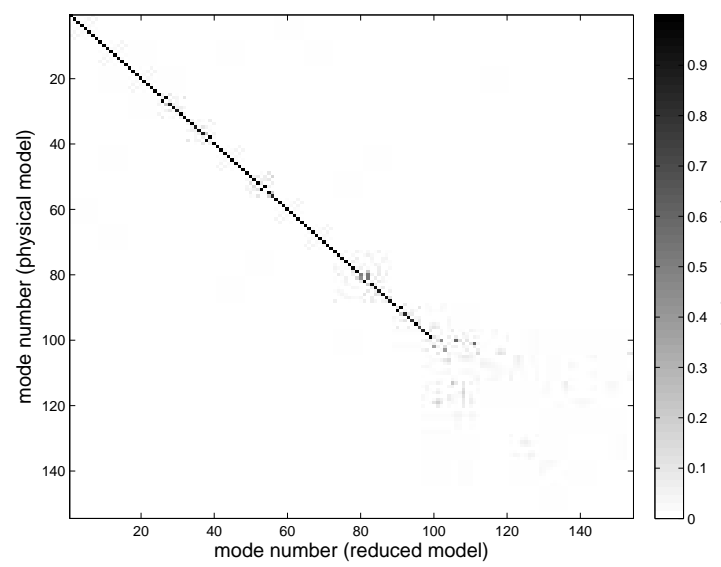

(A)

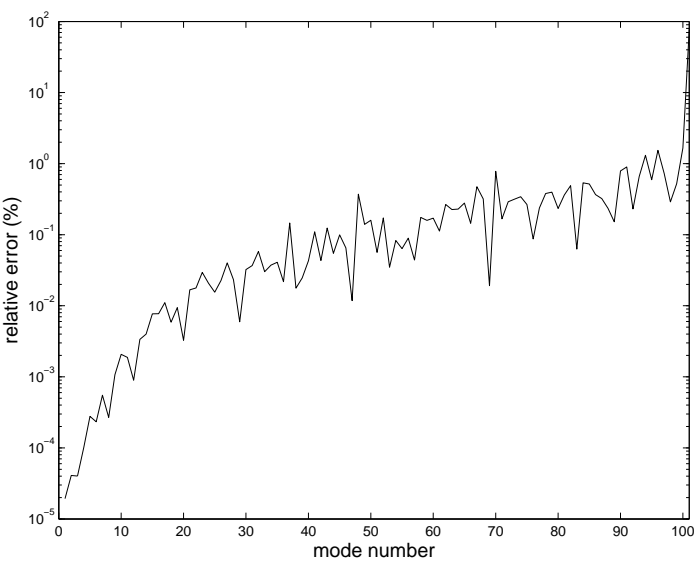

(в)

Figure 2: Comparison between the reduced and the physical model. (a): MAC criterion; (b): relative error

where $\mathbf{M}, \mathbf{C}$ and $\mathbf{K}$ are mass, damping and stiffness matrices. $\mathbf{X}$ is the generalized displacement vector and the dot denotes derivative with respect to the time. The vector $\mathbf{F}$ corresponds to the force due to the brake pressure applied over the back-plate of the pad. $\mathbf{F}_{\mathbf{N L}}$ defines the global non-linear force vector which contains linear and non-linear parts of the contact force vector and also the friction force vector for both disc and pad. Moreover, the contribution of the radial component of the friction force is neglected in this paper. It can be noticed two kinds of non-linearities in this contact formulation: the cubic contact law and the contact loss of contact configurations.

In this study, damping is equally distributed on the two modes involved in a mode coupling phenomenon (one of two modes being stable and the other being unstable). This configuration is chosen in order to be in the configuration of "equally damped coalescence" [18]. As previously explained in $[8,9,18]$, the only effect of adding equal damping on these modes is a shift of the curves towards the negative real parts (i.e. "damping always stabilizes the system"). So the modal damping rate $\zeta_{i}$ is equal to 10 for all the modes involved in a mode coupling phenomena. The notion of stability (i.e. stable and unstable modes) will be defined in section 3. For other modes, we consider a classical damping percentage $\xi_{i}=1 \%$ associated to the eigenfrequency $\omega_{i}$.

Thereby, the modal damping matrix $\mathbf{D}$ is used in order to compute $\mathbf{C}$. This matrix $\mathbf{D}$ is defined, in the modal base, as followed

$$
\mathbf{D}_{i i}= \begin{cases}\zeta_{i} & \text { if } \Phi_{i} \text { is associated with a mode coupling phenomenon } \\ 2 \xi_{i} \omega_{i} & \text { otherwise }\end{cases}
$$

Hence, the expression of the damping matrix $\mathbf{C}$ is given by

$$
\mathrm{C}=\boldsymbol{\Phi}^{-\mathbf{1}^{\mathrm{T}}} \mathbf{D} \Phi^{-1}
$$

where $\boldsymbol{\Phi}$ denotes the eigenvectors matrix of the reduced system. It can be noticed that the square matrix $\boldsymbol{\Phi}$ is obtained by:

$$
\left(\mathbf{K}-\omega^{2} \mathbf{M}\right) \Phi=\mathbf{0}
$$

where $\boldsymbol{\omega}$ is the diagonal matrix which contains all the eigenpulsations of the reduced system. 


\subsection{Boundary element model}

In this section the boundary element model developed is presented. This model is used to compute the sound pressure radiated by the brake system subjected to friction-induced nonlinear vibrations. For more details about the Boundary Elements Method, one can refer to [19]. The sound pressure radiated in the free space can be computed by solving the KirchhoffHelmholtz equation which is defined by

$$
\Delta P(\mathbf{r})+k^{2} P(\mathbf{r})=0
$$

where $\mathbf{r}$ denotes an exterior point of the brake system (coordinates of $M^{\prime}$ in Fig. 3), $P(\mathbf{r})$ is the outside sound pressure radiated and $k$ is the wave number defined by $k=\omega / c$ with $\omega$ the wave pulsation and $c$ the speed of sound in dry air. The boundary element method is based on the Kirchhoff-Helmholtz equation. The main feature of this method is the fact that the KirchhoffHelmholtz integral is calculated over a surface instead of a volume. It can be shown that the Kirchhoff-Helmholtz integral is of the following form

$$
\begin{gathered}
\epsilon P(\mathbf{r})=\int_{S}\left(P\left(\mathbf{r}^{\prime}\right) \frac{\partial G\left(\mathbf{r}-\mathbf{r}^{\prime}\right)}{\partial n}-G\left(\mathbf{r} \mid \mathbf{r}^{\prime}\right) \frac{\partial P\left(\mathbf{r}^{\prime}\right)}{\partial n}\right) d S \\
\epsilon=\left\{\begin{array}{cl}
1 & \text { for } \mathbf{r} \in V_{\text {ext }} \\
\frac{1}{2} & \text { for } \mathbf{r} \in S
\end{array}\right.
\end{gathered}
$$

where $\mathbf{r}^{\prime}$ defines a point over the surface $S$ (coordinates of $M$ in Fig. 3), $G$ is the Green function, $S$ is the surface that defines the skin of the brake system and $V_{\text {ext }}$ is the outside volume.

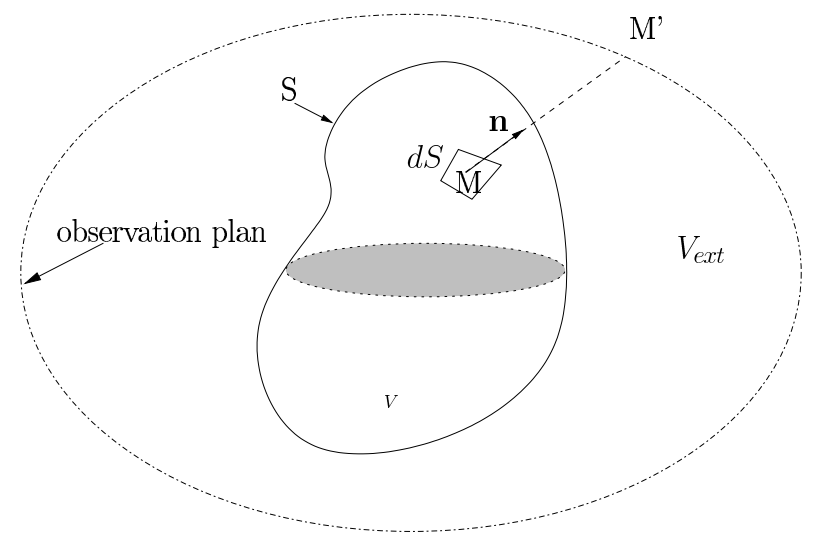

FIGURE 3: Illustration of the Kirchhoff-Helmholtz integral

The first level of approximation in the BEM is geometrical. Actually, the surface $S$ is split in $N_{e}$ subdivisions such as $\sum_{j=1}^{N_{e}} S_{j} \approx S$. The second kind of approximation used in this paper consists in assuming $P$ and $G$ to be constant in each element. The discrete Kirchhoff-Helmholtz integral takes the following expression

$$
\epsilon P(\mathbf{r})=\sum_{j=1}^{N_{e}} P\left(r_{j}^{\prime}\right) \int_{S_{j}} \frac{\partial G\left(\mathbf{r} \mid r_{j}^{\prime}\right)}{\partial n} d S_{\mathbf{r}^{\prime}}-\sum_{j=1}^{N_{e}} \frac{\partial P\left(r_{j}^{\prime}\right)}{\partial n} \int_{S_{j}} G\left(\mathbf{r} \mid r_{j}^{\prime}\right) d S
$$

that is to say

$$
\epsilon P\left(r_{i}\right)=\sum_{j=1}^{N_{e}} P\left(r_{j}^{\prime}\right) \mathbb{M}_{i j}-\sum_{j=1}^{N_{e}} \frac{\partial P\left(r_{j}^{\prime}\right)}{\partial n} \mathbb{L}_{i j}
$$


which can be written as

$$
\epsilon \mathbf{P}=\mathbb{M} \mathbf{P}_{\mathbf{S}}-\mathbb{L} \frac{\partial \mathbf{P}_{\mathbf{S}}}{\partial n}
$$

where $\mathbf{P}_{\mathbf{S}}$ corresponds to the sound pressure over the surface $S$, and $\mathbf{P}$ is the sound pressure in $V_{\text {ext }}$. The field of normal speed over $S$ is known in the acoustic problem so that $\frac{\partial \mathbf{P}_{\mathbf{S}}}{\partial n}$ is known. The boundary element method is applied by keeping the upper surface of the disc without the friction interface and the upper surface of the pad (see Fig. 4), and it contains about 3000 degrees of freedom. Actually, the friction interface is in contact with the fluid only during loss of contact configurations. So, it can be assumed that this area does not radiate. Moreover, the gap between the two previous surfaces contain fluid. So, acoustic interactions can occur: for example the pad pressure can be reflected over the disc. To avoid this coupling, pressure fields over the disc and the pad will be calculated separately. Into the exterior domain, radiations of both pad and disc will be considered. For this study, parts of the Open BEM toolbox, originally written by Peter Juhl and Vicente Cutanda Henriquez, are used (see [20]). In this toolbox, the authors use a Green function in free space defined by:

$$
G\left(\mathbf{r} \mid \mathbf{r}^{\prime}\right)=\frac{e^{-j k R}}{R}
$$

where $R=\left|\mathbf{r}-\mathbf{r}^{\prime}\right|$ denotes the distance between $M$ and $M^{\prime}$ and $j$ is such as $j^{2}=-1$.

According to Eq. (9), it is important to notice that the sound pressure $P$ depends on the position $\mathbf{r}$ but also on the wave number $k$. That is to say $P$ also depends on the wave frequency. The wave frequency $\omega$ cannot be predicted for self-excited system and this point will be developed further.

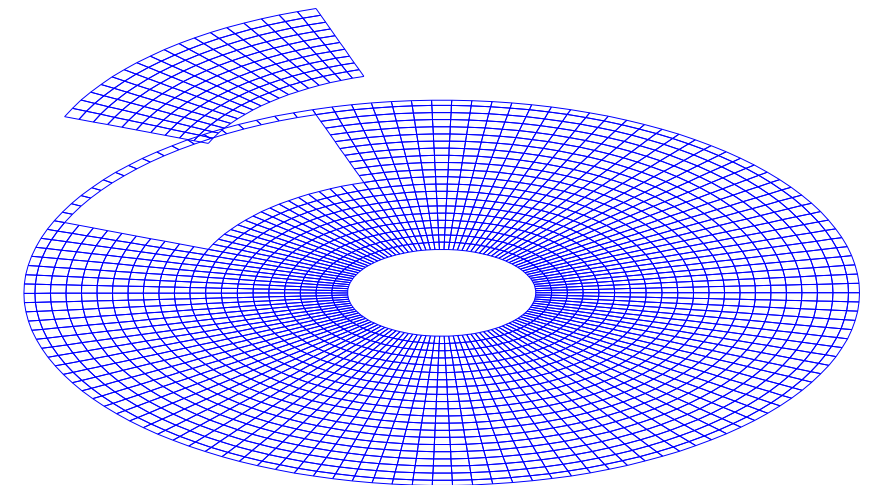

Figure 4: Boundary element model of the brake system.

\section{$3 \quad$ Stability analysis}

What we call "system instability" is the divergence of a sliding equilibrium point of the brake system due to friction-induced vibrations. For this reason, a set of parameters $\left(k_{L}, k_{N L}\right.$ and $\left.F\right)$ has to be defined and then the stability of the sliding equilibrium points has to be estimated for each friction coefficient $\mu$. The stability of the previous non-linear brake system is performed by following three steps:

step 1: Computation of the static sliding solution $\mathbf{X}_{\mathbf{0}}$ 
$\mathbf{K X}_{\mathbf{0}}=\mathbf{F}_{\mathrm{NL}}+\mathbf{F}$

step 2: Linearization around $\mathbf{X}_{0}: \quad \mathbf{X}=\mathbf{X}_{0}+\tilde{\mathbf{X}}$

$\mathbf{M} \ddot{\tilde{\mathbf{X}}}+\mathbf{C} \dot{\tilde{\mathbf{X}}}+\left(\mathbf{K}-\mathbf{J}_{\mathbf{N L}, \mathbf{X}_{\mathbf{0}}}\right) \tilde{\mathbf{X}}=\mathbf{0}$

step 3: Complex eigenvalue analysis of the linearized system

$\left(\lambda^{\mathbf{2}} \mathbf{M}+\lambda \mathbf{C}+\left(\mathbf{K}-\mathbf{J}_{\mathbf{N L}, \mathbf{X}_{\mathbf{0}}}\right)\right) \mathbf{\Phi}=\mathbf{0}$

where $\tilde{\mathbf{X}}$ is the disturbance of the equilibrium point $\mathbf{X}_{\mathbf{0}}$, and $\mathbf{J}_{\mathrm{NL}}$ corresponds to the linearized expression of the non-linear force vector $\mathbf{F}_{\mathrm{NL}}$ which is composed of friction and contact forces. $\mathbf{F}_{\mathbf{N L}}$ is approximated by a one order Taylor development around $\mathbf{X}_{\mathbf{0}}$ that is to say

$$
\left.\mathbf{F}_{\mathbf{N L}}(\tilde{\mathbf{X}}) \approx \sum_{i} \frac{\partial \mathbf{F}_{\mathbf{N L}}(\tilde{\mathbf{X}})}{\partial \tilde{\mathbf{X}}_{i}}\right|_{\mathbf{X}_{\mathbf{0}}} \tilde{\mathbf{X}}_{i}=\mathbf{J}_{\mathbf{N L}, \mathbf{X}_{\mathbf{0}}} \tilde{\mathbf{X}}
$$

Then, step 3 provides the stability of the sliding equilibrium. If $\mu$ is a positive scalar, $\mathbf{J}_{\mathbf{N L}, \mathbf{X}_{\mathbf{0}}}$ is non-symmetric, so the eigenvalues $\lambda_{i}$ are complex. If all the real parts of the eigenvalues are negative, the sliding equilibrium point $\mathbf{X}_{\mathbf{0}}$ is stable. If at least one of the eigenvalues has a positive real part, an instability can be generated for the equilibrium point $\mathbf{X}_{\mathbf{0}}$ and this unstable configuration can lead to self-excited oscillations. It is important to notice that the Lyapunov theorem can be applied and all the previous conclusion about stability can be extended from the linearized system to the non-linear one. For the brake system under study, the sets of parameters defined in tables 1 and 2 are used.

\begin{tabular}{lcccc}
\hline Property & & & Disc & Pad \\
\hline Young's modulus & $E$ & $\mathrm{GPa}$ & 125 & 2 \\
volumic density & $\rho$ & $\mathrm{kg} \cdot \mathrm{m}^{-3}$ & 7200 & 2500 \\
Poisson's ratio & $\nu$ & & 0.3 & 0.1 \\
Inner radius & $R_{\text {inner }}$ & $\mathrm{cm}$ & 3.4 & 9.1 \\
Outer radius & $R_{\text {outer }}$ & $\mathrm{cm}$ & 15.1 & 14.7 \\
Thickness & $t$ & $\mathrm{~cm}$ & 1.9 & 1.28 \\
Pad angle & $\theta$ & $\circ$ & - & 50 \\
\hline
\end{tabular}

TABLE 1: Material and geometrical properties of the brake system

\begin{tabular}{lcc}
\hline \multicolumn{3}{l}{ Model parameters } \\
\hline$k_{L}$ & $9 e^{4}$ & $\mathrm{Nm}^{-1}$ \\
$k_{N L}$ & $4 e^{9}$ & $\mathrm{Nm}^{-3}$ \\
$F$ & 70 & $\mathrm{~N} /$ nodes \\
$\xi$ & 1 & $\%$ \\
$\zeta_{i}$ & 10 & \\
\hline
\end{tabular}

TABLE 2: Model parameters: linear and cubic contact stiffness $k_{L} k_{N L}$, braking force per nodes $F$, damping parameters $\xi$ and $\zeta_{i}$

Fig. 6 shows the complex eigenvalues analysis as a function of the friction coefficient $\mu$. The first instability is detected for a friction value of $\mu=0.7$ (Fig. 6a) and the associated frequency 


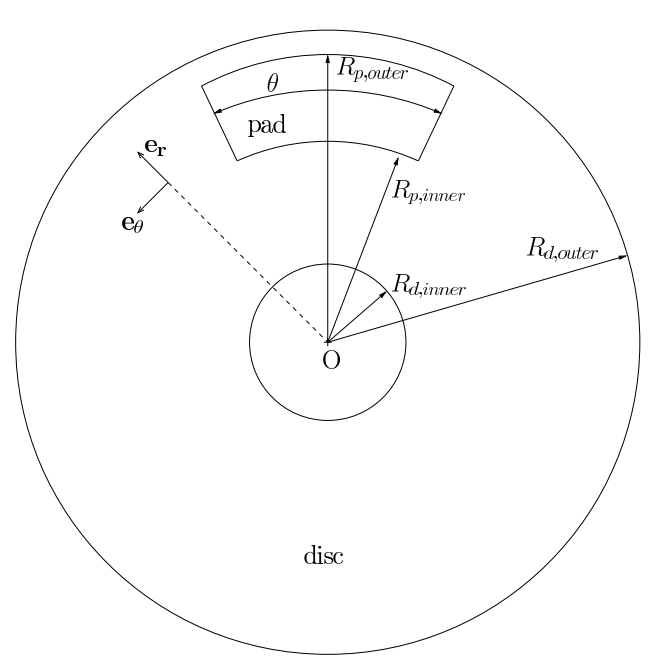

FiguRE 5: Illustration of the geometrical parameters

is $929.8 \mathrm{~Hz}$, as indicated in Fig. 6b. The second instability occurs for a friction coefficient of $\mu=0.73$ (Fig. 6c). The associated unstable mode is detected at $9418 \mathrm{~Hz}$ (Fig. 6d). It can be noticed that by increasing the friction coefficient, the number of unstable modes increases as well.

\section{Non-linear vibrations and calculation of the radiated sound pressure}

In this section, a particular attention is drawn on the sound pressure radiated in the free space due to the non-linear vibrations of the brake system. These non-linear vibrations are due to the frictional interface that generates self-excited vibrations. In order to be able to numerically quantify this sound pressure, a method which couples non-linear vibrations and the radiated sound pressure is proposed.

The cases under study are listed in Table 3. As explained previously, stability analysis is not

\begin{tabular}{cccc}
\hline Case & Friction coefficient & Frequency $f_{1}(\mathrm{~Hz})$ & Frequency $f_{2}(\mathrm{~Hz})$ \\
\hline 1 & 0.72 & 929.8 & - \\
2 & 0.74 & 930.3 & 9418 \\
\hline
\end{tabular}

TABLE 3: List of cases under study

sufficient to predict the frequencies of the non-linear vibrations of the brake system. Actually, linearized equations are used to perform the complex eigenvalue analysis. So, the stability analysis is only valid locally near the static sliding equilibrium point. This can only predict all the unstable modes which could participate to the non-linear vibrations.

The solution of the equation of motion for self-excited system is composed of two regimes: transient non-linear vibrations with a divergence rate defined by the real part of the positive eigenvalues, then pseudo-periodic non-linear vibrations occur. It is interesting to evaluate the level and the map of sound pressure during these two regimes. So, these two regimes are studied and the Fourier transform is used to determine all the frequencies contained in the time history 


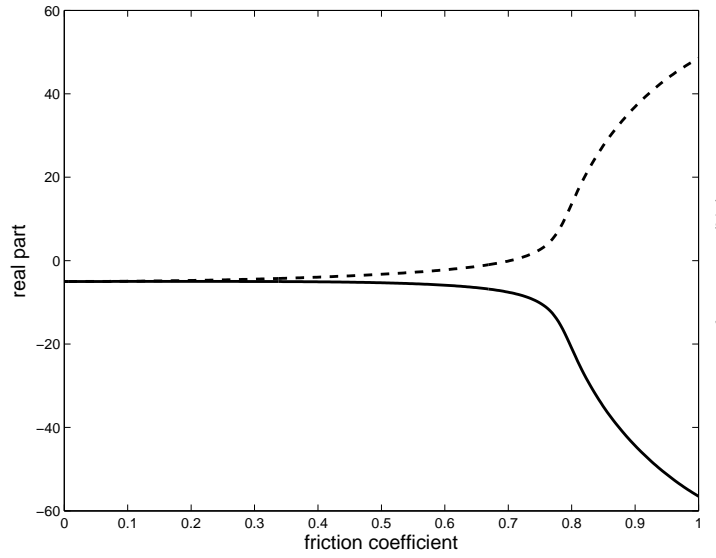

(A)

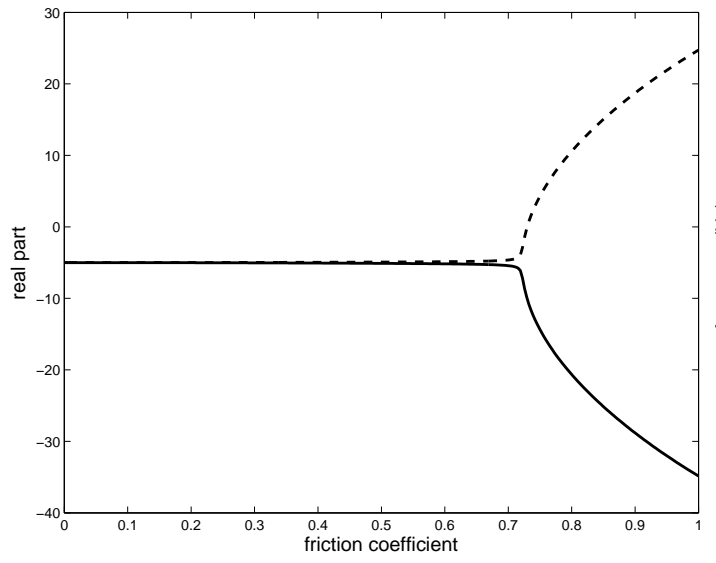

(c)

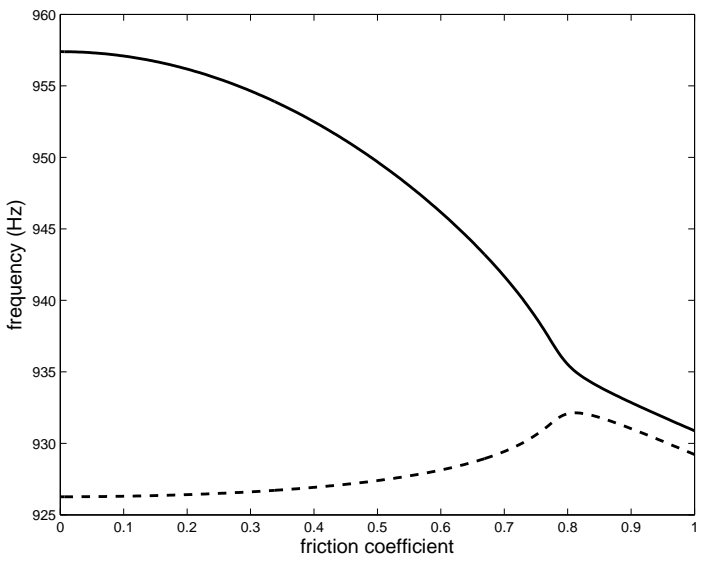

(в)

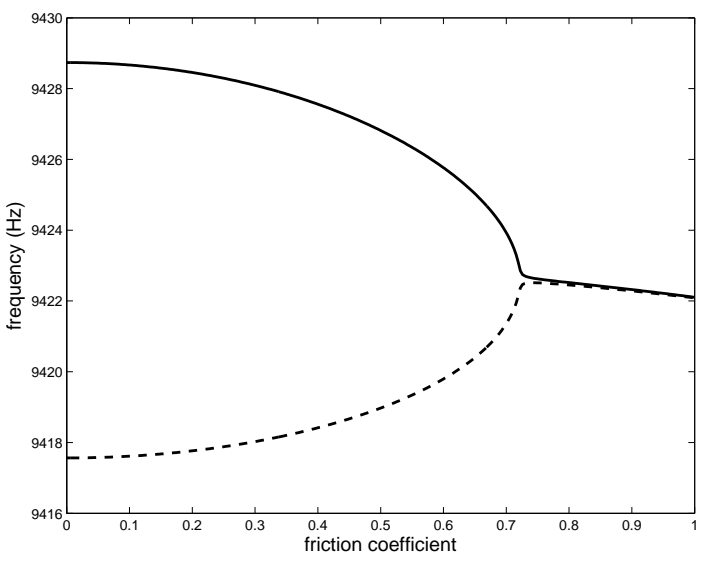

(D)

Figure 6: Stability analysis of the brake system. (a) (c): real parts; (b) (d): frequencies 
response. The aim of this operation is to isolate all the contributions of the velocity for each frequency. That is to say, to decompose the global wave in elementary waves defined by a single frequency. Then, the boundary element problem is solved for each elementary waves and the sound pressure associated to each waves can be computed. Finally, the global sound pressure is calculated by superposition. In the following, the complete process is explained and developed in details.

\subsection{Time integration}

The first step of this coupling is to solve the equation of motion (Eq. (5)) in order to compute the time history responses for both generalized displacements $\mathbf{X}(t)$ and velocity $\dot{\mathbf{X}}(t)$. In this aim, time integrations are performed by using a classical Runge-Kutta method. First, the order of Eq. (5) is reduced by introducing a state variable defined by $\mathbf{Y}=\left[\begin{array}{ll}\dot{\mathbf{X}} & \mathbf{X}\end{array}\right]^{T}$, where $\dot{\mathbf{X}}$ and $\mathbf{X}$ denotes the generalized velocity vector and displacement vector respectively. Then, the equation of motion (Eq. (5)) takes the following form

$$
\dot{\mathbf{Y}}=\mathbf{B Y}+\mathbf{E}^{-1}\left(\tilde{\mathbf{F}}+\tilde{\mathbf{F}}_{\mathbf{N L}}(\mathbf{Y})\right)
$$

where

$$
\begin{aligned}
& \mathbf{E}=\left[\begin{array}{cc}
\mathbf{M} & \mathbf{0} \\
\mathbf{0} & \mathbf{M}
\end{array}\right] \quad ; \quad \mathbf{H}=\left[\begin{array}{cc}
\mathbf{C} & \mathbf{K} \\
-\mathbf{M} & \mathbf{0}
\end{array}\right] \quad ; \quad \mathbf{B}=-\mathbf{E}^{-\mathbf{1}} \mathbf{H}
\end{aligned}
$$

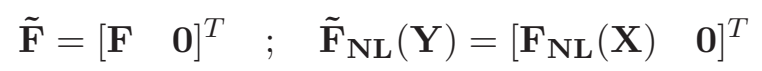

For all the cases under study, the time integration is initialized near the static sliding equilibrium point $\mathbf{X}_{\mathbf{0}}$. The dofs of $\mathbf{X}_{\mathbf{0}}$ associated to the unstable modes are disturbed. So, the initial vector is of the form $\mathbf{Y}(t=0)=\left[\begin{array}{ll}\mathbf{0} & \mathbf{X}_{\mathbf{0}}+\tilde{\mathbf{X}}\end{array}\right]^{T}$, where $\tilde{\mathbf{X}}$ denotes the introduced disturbance.

\subsection{Determination of the vibrations frequencies}

For one unstable mode, the frequencies involved are the fundamental frequency $f_{1}$ and its harmonic components, whereas for two unstable modes, the frequencies are of the form $\pm m f_{1} \pm n f_{2}$ where $f_{1}$ and $f_{2}$ are the fundamental frequencies [10]. The main difficulty of this issue is to determine all the couples $\left(m_{i}, n_{i}\right)$ which define all the combinations $m_{i} f_{1}+n_{i} f_{2}$, where $m_{i}$ and $n_{i}$ are positive or negative integers. So, the second step consists in applying Fourier transforms on each dofs of $\dot{\mathbf{X}}(t)$ and to determine all the couples $\left(m_{i}, n_{i}\right)$ by detecting the peaks of each Fourier transforms. This step provides all the couples $\left(m_{i}, n_{i}\right)$ and thus the frequencies $f_{n}=m_{n} f_{1}+n_{n} f_{2}$ which compose the spectrum of velocity.

\subsection{Decomposition by order}

Then, velocity vector $\dot{\mathbf{X}}(t)$ can be decomposed in a Fourier serie

$$
\dot{\mathbf{X}}(t)=\sum_{n=0}^{N_{\text {order }}} \mathbf{a}_{\mathbf{n}} \cos \left(2 \pi f_{n} t\right)+\mathbf{b}_{\mathbf{n}} \sin \left(2 \pi f_{n} t\right) \quad \text { with } \mathbf{b}_{\mathbf{0}}=\mathbf{0}
$$

where $\mathbf{a}_{\mathbf{n}}$ and $\mathbf{b}_{\mathbf{n}}$ are the Fourier coefficients computed for a period in the pseudo-periodic regime. $N_{\text {order }}$ is the highest order defined by $N_{\text {order }}=\max \left(\left|m_{n}\right|+\left|n_{n}\right|\right)$. However, as explained previously, all the components of the velocity have to be sorted by frequencies. The third step 
consists in building the Fourier base and project the velocity onto it. All the contributions of the velocity $\dot{\mathbf{X}}_{n}(t)$, sorted by order, can be obtained by

$$
\dot{\mathbf{X}}_{n}(t)=\mathbf{a}_{\mathbf{n}} \cos \left(2 \pi f_{n} t\right)+\mathbf{b}_{\mathbf{n}} \sin \left(2 \pi f_{n} t\right)
$$

and by superposition

$$
\dot{\mathbf{X}}(t)=\sum_{n=0}^{N_{\text {order }}} \dot{\mathbf{X}}_{n}(t)
$$

\subsection{Sound pressure radiated}

Finally, the sound pressure over the surface $S$ denoted by $\mathbf{P}_{\mathbf{S}_{\mathbf{n}}}$ can be calculated for each order. That is to say, the global wave is decomposed into elementary waves. $\mathbf{P}_{\mathbf{S}_{\mathbf{n}}}$ depends only on the velocity over $S$ in the normal direction denoted by $\dot{\mathbf{x}}_{S_{n}}(t)$. Then, the boundary element equation (Eq. (13)) can be solved for each order and it takes the following form

$$
\epsilon \mathbf{P}_{\mathbf{n}}=\mathbb{M} \mathbf{P}_{\mathbf{S}_{\mathbf{n}}}-\mathbb{L} \frac{\partial \mathbf{P}_{\mathbf{S}_{\mathbf{n}}}}{\partial n}
$$

where $\mathbf{P}_{\mathbf{n}}$ is the sound pressure in the free space associated to the order $n$. The global sound pressure can be obtained by

$$
\mathbf{P}=\sum_{n=0}^{N_{\text {order }}} \mathbf{P}_{\mathbf{n}}
$$

\section{$5 \quad$ Numerical study}

In this section the two cases defined in Table 3 are investigated. Time history responses are presented for single and multi-instabilities, then the sound pressure radiated is calculated for both cases.

\subsection{Time history responses}

\subsubsection{Case 1: single instability}

Case 1 is defined by a friction coefficient of $\mu=0.72$ and according to the stability analysis, this case could lead to self-excited vibrations composed of the fundamental frequency $f_{1}$ and its harmonics. Fig. 7 shows the time history response of a normal dof of the frictional interface. Actually, it can be observed that both displacement and velocity increase during a short transient regime, then a periodic non-linear regime is reached. Fig. 7c shows the limit cycles during the stationary regime and it can be noted that all the cycles are closed. The response is periodic. Fig. $7 \mathrm{~d}$ shows that the time history response contains the fundamental frequency $f_{1}$ and its harmonic components, such as $2 f_{1}$. The fundamental frequency $f_{1}$ detected is the same as the frequency predicted by the stability analysis. The appearances of harmonic components are due to non-linear effects of both loss of contact and non-linear contact stiffness. Spectrum analysis performed for the velocity along $x$ - and $y$-directions show the same harmonic components. It is important to notice that the stability analysis cannot predict the presence of the second harmonic component $2 f_{1}$ for the transient and stationary responses. 


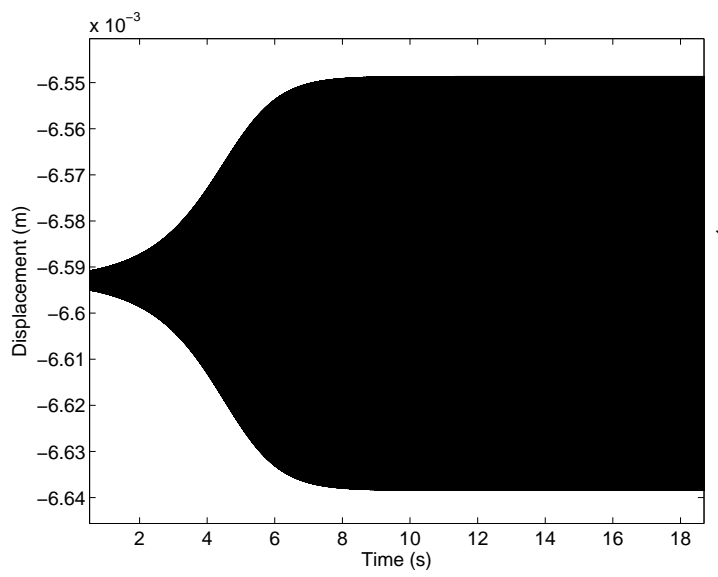

(A)

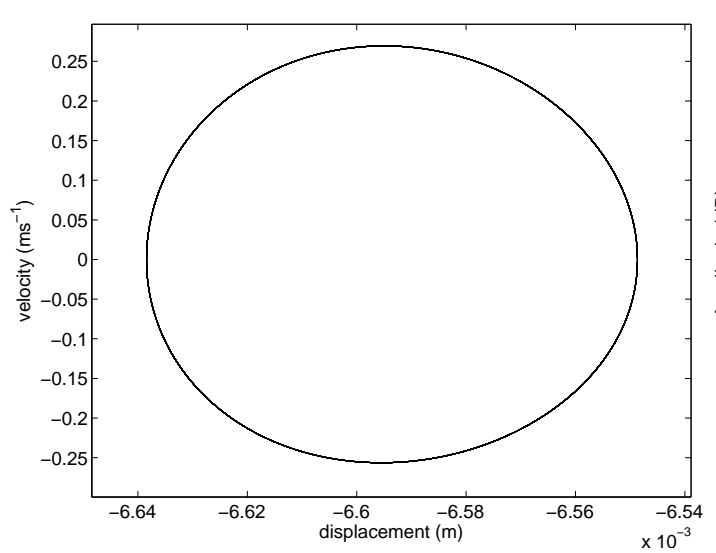

(c)

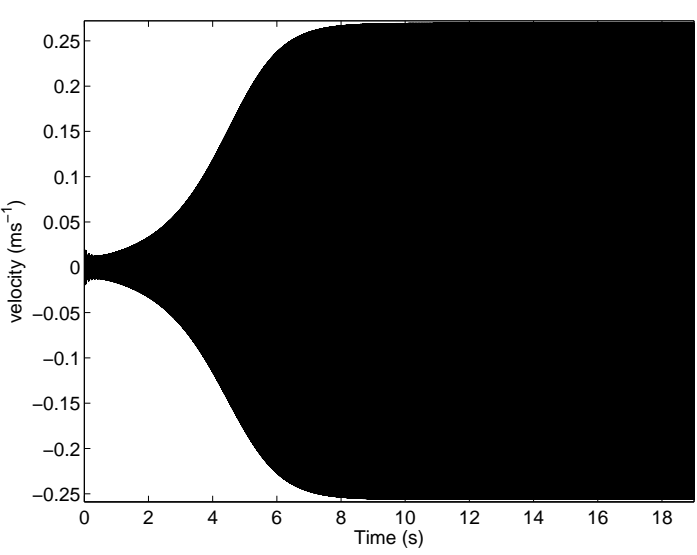

(B)

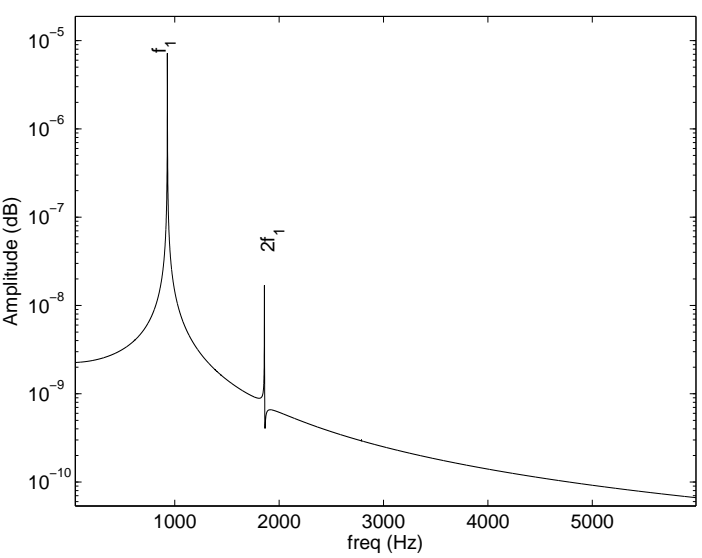

(D)

FIGURE 7: Time history response for case 1: $\mu=0.72$. (a) displacements along $\mathbf{z}$-direction respectively; (b) velocity along z-direction respectively; (c) limit cycles; (d) Fourier transform 


\subsubsection{Case 2: multi-instabilities}

As explained in section 3, the brake system can be affected by two instabilities (i.e. two mode coupling phenomena) for a friction coefficient greater than $\mu>0.73$. Fig. 8 illustrates the nonlinear transient and stationary oscillations of the brake system for a dof of the interface between the disc and the pad (for a friction coefficient of 0.74). Fig. 8a-c shows the displacements of a node at the friction interface in the three directions. The divergence rate is higher than case 1 and so are the amplitudes. Moreover, a transient regime occurs for $t \in[0 ; 4]$ (see zone A in Fig. 8a) until a stationary regime is reached for $t \in[4 ; 18]$ (see zone B in Fig. 8a).

To characterize the response during these two regimes, the limit cycles have to be analyzed and a spectrum analysis has to be performed for both zones A and B. Involving the transient regime (zone A), Fig. 9a-c shows the associated limit cycles: they are very different from case 1. Actually, the cycles are not closed and this behaviour is due to the presence of two fundamental frequencies: the response is quasi-periodic (instead of being periodic in case 1). The spectrum of the velocity is then computed and Fig. 9d-f confirm the presence of two fundamental frequencies. The two detected fundamental frequencies are $f_{1}=930 \mathrm{~Hz}$ and $f_{2}=9423 \mathrm{~Hz}$, whereas the stability analysis predicts $f_{1}=929.8 \mathrm{~Hz}$ and $f_{2}=9418 \mathrm{~Hz}$. As previously explained in [10], this difference can be due to the fact that linear conditions (i.e. the linearized stability around an initial equilibrium point) are not valid during transient oscillations. Moreover, the case of multiinstabilities shows the appearance of harmonic components defined by $m_{i} f_{1}+n_{i} f_{2}$ as explained previously. The Fourier transforms show the following harmonic components: $f_{1}, 2 f_{1}, 3 f_{1}, 4 f_{1}$, $-2 f_{1}+f_{2},-f_{1}+f_{2}, f_{2}, f_{1}+f_{2}, 2 f_{1}+f_{2}, \ldots$, all these harmonic components are listed in Table 5. During the stationary regime defined by zone B, the final limit cycles are very different from those in zone A. Actually, the profile of the limit cycles are very different from those during the transient and the amplitudes are significantly more important as showed in Fig. 10a-c. Moreover, the Fourier transforms show the appearance of new harmonic components in the velocity spectrum. As previously showed, two fundamental frequencies are detected: $f_{1}=918$ $\mathrm{Hz}$ and $f_{2}=9424 \mathrm{~Hz}$ and these frequencies are still different from the prediction of the stability analysis. All the harmonic components detected are listed in Table 6.

For both cases 1 and 2, spectrum analysis shows the appearance of peaks which are not predicted by the stability analysis. Moreover, a variation of the fundamental frequencies is observed and as previously explained in [10], this evolution is due to a variation of the sliding equilibrium point during the braking process. These two previous notes show the necessity to perform a non-linear analysis which complete the stability analysis. Actually, we recall that stability analysis is the first step in the study of friction-induced vibration problems but it only gives information about initial divergence rate of disc brake amplitudes.

\subsection{Sound pressure radiated by brake squeal phenomenon}

In this section, the focus is on the sound pressure radiated by the brake system during selfexcited vibrations. The two previously presented cases (cases 1 and 2) are studied by applying the method proposed in section 4. Noise emissions will be investigated by investigating the acoustic intensity in decibels $L_{d B}$ defined by:

$$
L_{d B}=10 \log _{10}\left(\frac{\mathbf{P P}^{*}}{P_{r e f}^{2}}\right)
$$

where $P_{r e f}$ denotes the minimum audible sound pressure $\left(P_{r e f}=2 e^{-5} \mathrm{~Pa}\right)$.

In a first time, three observation planes are used: the first is the boundary element mesh and it permits to observe sound propagation directly over the brake system surface; the second 


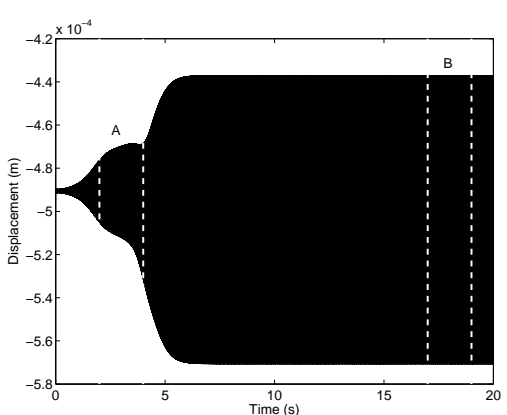

(A)

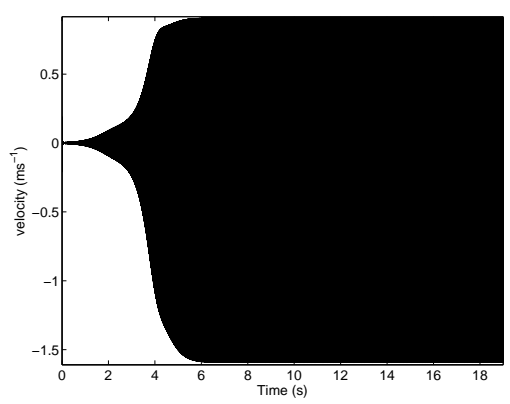

(D)

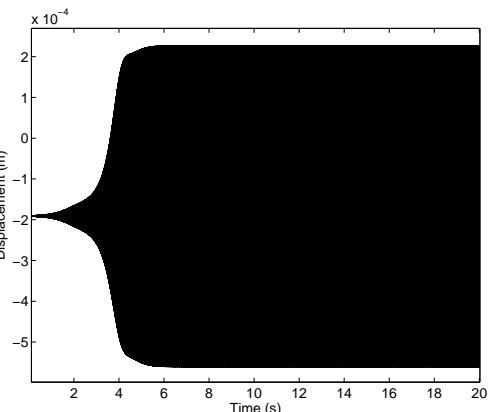

(в)

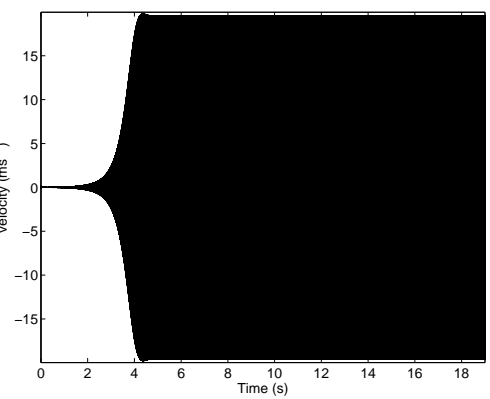

$(\mathrm{E})$

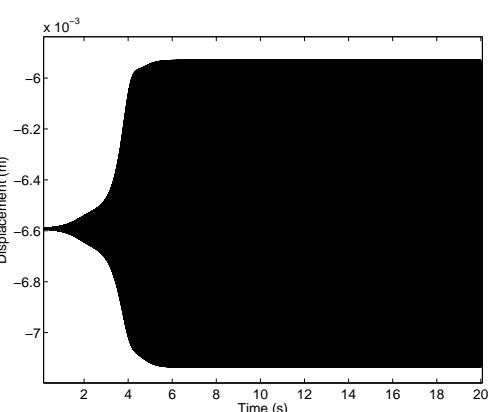

(c)

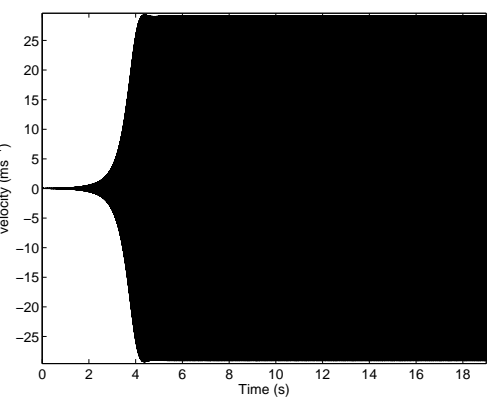

$(\mathrm{F})$

Figure 8: Time history response for case 2: $\mu=0.74$. (a) (b) (c) displacements along $\mathbf{x}-\mathbf{y}$ and $\mathbf{z}$-directions respectively; (d) (e) (f) velocity along $\mathbf{x}-\mathbf{y}$ - and $\mathbf{z}$-directions respectively

is a square of $1.5 \times 1.5 \mathrm{~m}$, centred over the disc, placed at $5 e^{-2} \mathrm{~m}$ high; the third is a square of $4 \times 4 \mathrm{~m}$, centred over the disc, placed at $1 \mathrm{~m}$ high. The acoustic intensity is displayed over these three planes in order to present the evolution of the map pressure as a function of high. That is to say, to show the near field and the far field acoustic intensity. Moreover, the directivity pattern is investigated over four other planes denoted by $P_{\theta}$ with $\theta=0, \frac{\pi}{2}, \frac{\pi}{4}, \frac{3 \pi}{4}$, which are squares of $1.5 \times 1.5 \mathrm{~m}$ (for $\theta=0, \frac{\pi}{2}$ ) and $1.5 \sqrt{2} \times 1.5 \mathrm{~m}$ (for $\theta=\frac{\pi}{4}, \frac{3 \pi}{4}$ ) in the planes defined by $\left(\mathbf{x}_{\theta}, \mathbf{z}\right)$ (see Fig. 11).

As explained in section 2.2, sound pressure calculation over the boundary element mesh is carried out by avoiding acoustic interactions between the disc and the pad. It is important to note that over the six previous planes, the global sound pressure composed of both disc and pad radiations is investigated.

Finally, the acoustic response is calculated for only one time step which corresponds to the maximum amplitude of the associated field of velocity.

\subsubsection{The case of single instability}

For the case $1(\mu=0.72)$, Fourier transforms of the velocity are performed for all the dofs. In this case, only one fundamental frequency $f_{1}$ is detected and it is the same as the stability analysis prediction: $f_{1}=930 \mathrm{~Hz}$. By applying the method detailed in sections 4.2 and 4.3 , all the frequencies which compose the field of velocity are detected (see Fig. 7d). These frequencies are listed in Table 4 and only the fundamental frequency $f_{1}$ and several harmonic components are present. All the harmonic components are retained for the calculation of the pressure. 


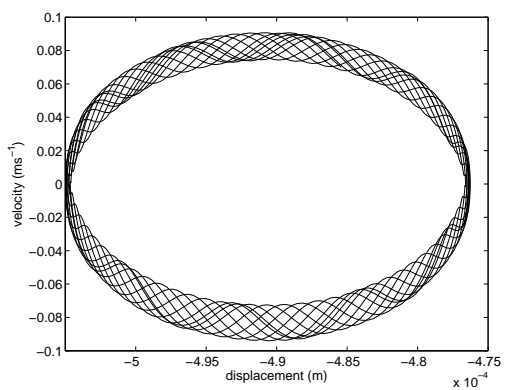

(A)

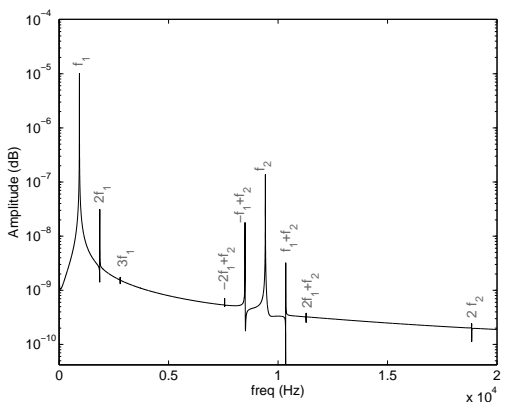

(D)

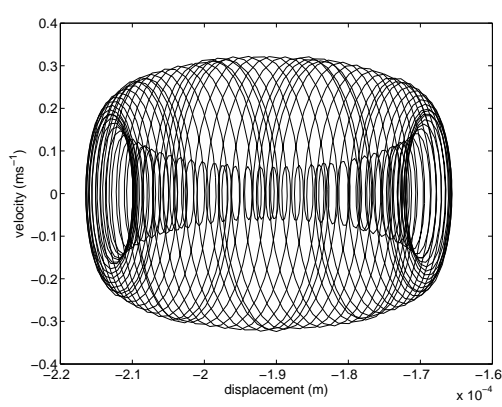

(в)

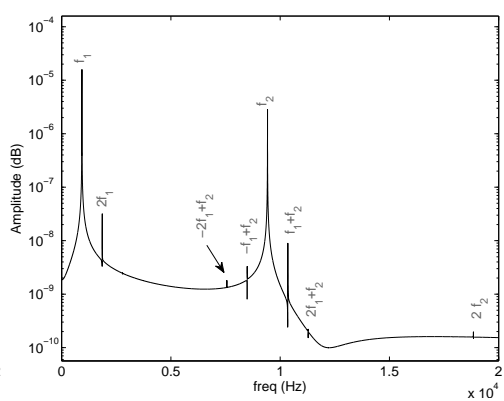

(E)

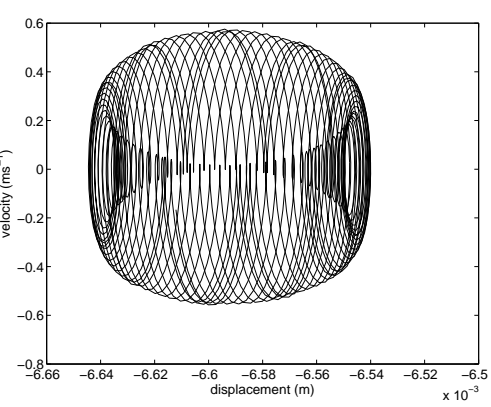

(c)

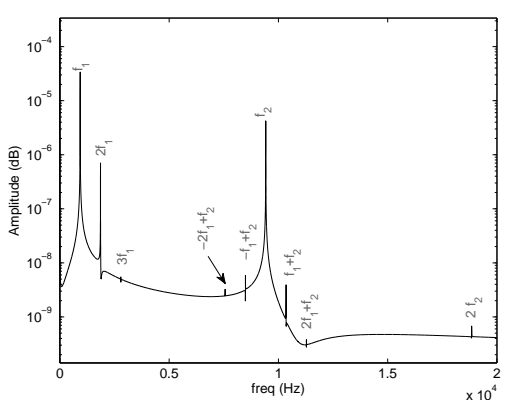

$(\mathrm{F})$

Figure 9: Spectrum analysis during transient (zone A) for case 2: $\mu=0.74$. (a) (b) (c) limit cycles in zone A along $\mathbf{x}-\mathbf{y}$ - and $\mathbf{z}$-directions respectively; (d) (e) (f) Fourier transforms in zone A

\begin{tabular}{ccc}
\hline order & harmonic component & frequency $(\mathrm{Hz})$ \\
\hline 1 & $f_{1}$ & 930 \\
2 & $2 f_{1}$ & 1860 \\
3 & $3 f_{1}$ & 2790 \\
4 & $4 f_{1}$ & 3720 \\
5 & $5 f_{1}$ & 4650 \\
6 & $6 f_{1}$ & 5580 \\
10 & $10 f_{1}$ & 9300 \\
\hline
\end{tabular}

TABle 4: Detected frequencies for case 1: $\mu=0.72$ 


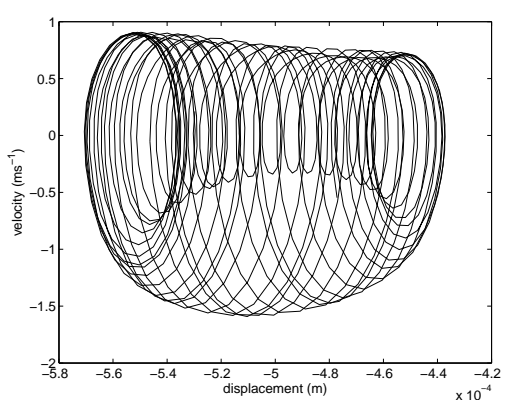

(A)

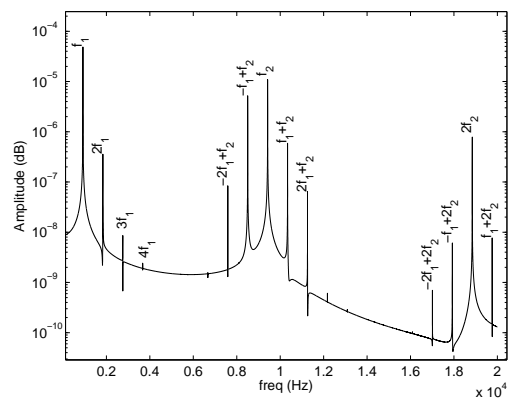

(D)

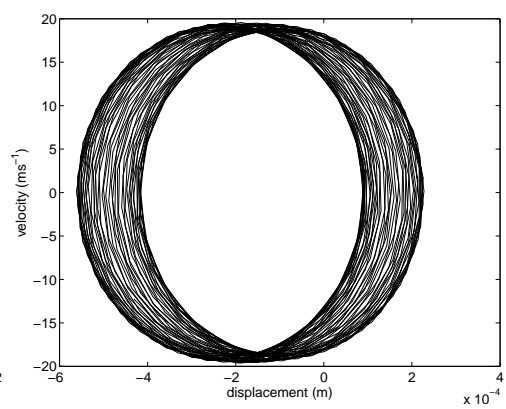

(B)

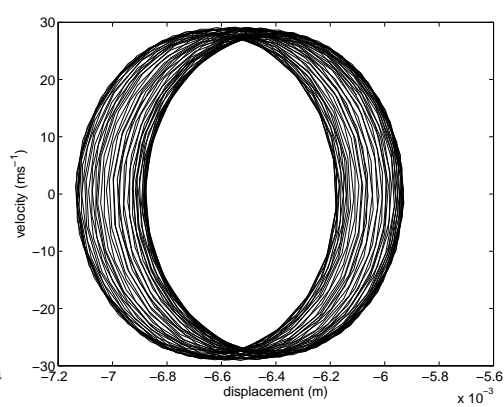

(C)

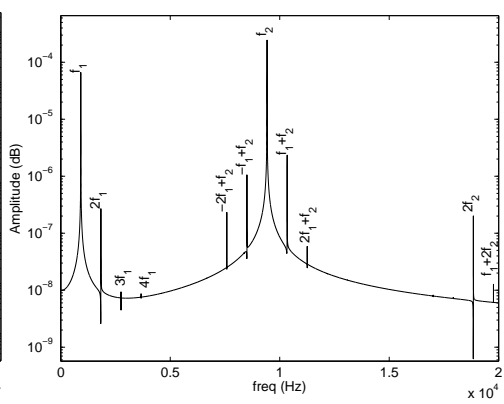

(E)

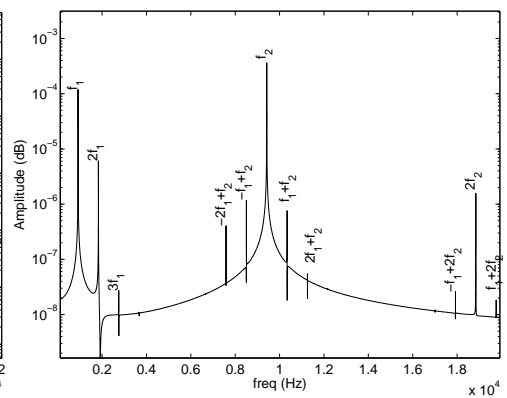

(F)

Figure 10: Spectrum analysis during stationary regime (zone $B$ ) for case 2: $\mu=0.74$. (a) (b) (c) limit cycles in zone $B$ along $\mathbf{x}-\mathbf{y}$ - and $\mathbf{z}$-directions respectively; (d) (e) (f) Fourier transforms in zone $B$

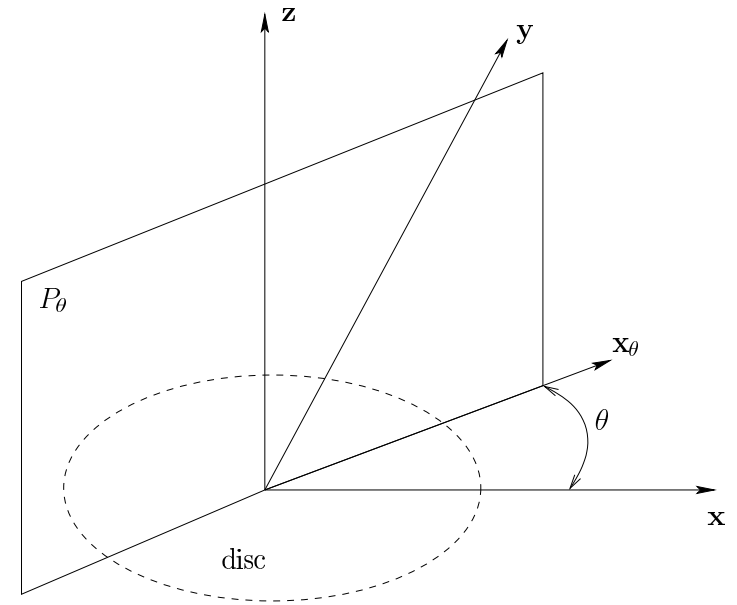

(A)

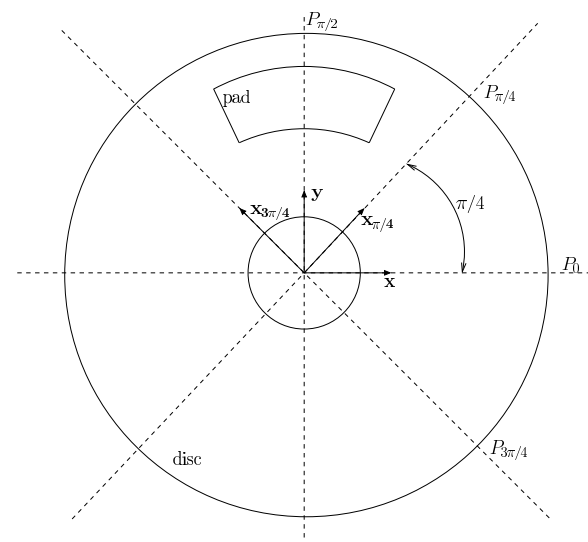

(B)

Figure 11: (a) definition of the observation plan $P_{\theta}$; (b) observation planes $P_{\theta}$ used: $P_{0}, P_{\frac{\pi}{2}}$, $P_{\frac{\pi}{4}}$ and $P_{\frac{3 \pi}{4}}$ 
As explained in section 4.4, the sound pressure radiated is calculated and the acoustic intensity is displayed over the three horizontal surfaces previously defined. Fig. 12 shows the acoustic intensity $L_{d B}$ radiated during stationary regime for case 1 . Fig. 12a corresponds to the intensity over the boundary element mesh. It can be seen that pad levels are between 100 and $124 \mathrm{~dB}$ and disc levels are between 93 and $115 \mathrm{~dB}$. Moreover, it can be noted that the system presents five areas of maximum intensity: two over the pad and three over the disc. Involving near field global radiations, Fig. 12b shows the appearance of four lobes of directivity. Three of them are predominants and correspond to the predominant areas of maximum intensity over the disc and the pad. The less predominant lobe is due to the less predominant area of acoustic intensity of the disc. Levels in near field go from 61 to $127 \mathrm{~dB}$ and the maximum value of $L_{d B}$ is above the pad. Fig. 12c corresponds to the far field acoustic intensity and it can be seen the presence of only two main lobes: the lobes in near field disappear with the distance from the source. Levels in far field go from 21 to $82 \mathrm{~dB}$. The three previous planes permit to conclude that levels of intensity decrease with the distance from the source, this validates the classical evolution of the

sound pressure: in near field the level decreases as $\frac{1}{R^{2}}$ and in far field, it decreases as $\frac{1}{R}$. Moreover, the directivity patterns also change. Involving vertical observation planes $P_{\theta}$, it can be seen that over $P_{0}$ the intensity presents one main lobe: the propagation is mainly unidirectional (see Fig. 15a). Over $P_{\pi / 4}$, the previous lobe is present but two other lobes appear (see Fig. 15c). Finally, over $P_{\pi / 2}$ and $P_{3 \pi / 4}$, the propagation is omni-directional as illustrated in Fig. 15e and g.

\subsubsection{The case of multi-instabilities}

For case $2(\mu=0.74)$, time history analysis lets appear two peculiar regimes: a transient defined by zone $\mathrm{A}$ and a stationary defined by zone $\mathrm{B}$ as indicated in Fig. 8. For the case of multiinstabilities under study, a comparison between the sound pressure radiated in these two regimes is carried out.

Sound pressure in transient regime As explained previously, the multi-instabilities case presents a peculiar transient regime. For this regime, a spectrum analysis is performed and all the frequencies which composed the time responses are detected. All these frequencies are listed in Table 5 and the method proposed in section 4.2 and 4.3 is applied by keeping all the harmonic components.

Fig. 13 shows the acoustic radiation for the two unstable modes case during zone A. Fig. 13a shows the intensity $L_{d B}$ over the boundary element mesh. It can be noticed that levels go from 60 to $93 \mathrm{~dB}$ over the disc and from 90 to $129 \mathrm{~dB}$ over the pad. Moreover, the acoustic intensity over the pad presents several local maximums and over the disc it presents a large area of maximum intensity. In near field (Fig. 13b), levels are between 56 and $127 \mathrm{~dB}$ and in far field (Fig. 13c), $L_{d B}$ goes from 10 to $72 \mathrm{~dB}$. In near and far fields, the directivity pattern is composed of four lobes. It can be seen that there contours are not smooth due to the fact that during zone A, squeal is not well established.

Sound pressure in stationary regime During the stationary regime (zone B), Fourier transforms of the velocity are calculated for all the dofs. In this case, two fundamental frequencies $f_{1}$ and $f_{2}$ are detected. The first fundamental frequency $f_{1}=944 \mathrm{~Hz}$ is different from the stability analysis prediction of $930.2 \mathrm{~Hz}$ whereas the second fundamental frequency $f_{2}$ remains the same. By applying points 2 and 3 detailed in section 4, the frequencies which compose the field of velocity (see Fig. 8d-f) are detected. These frequencies are listed in Table 6 and it can be noticed 


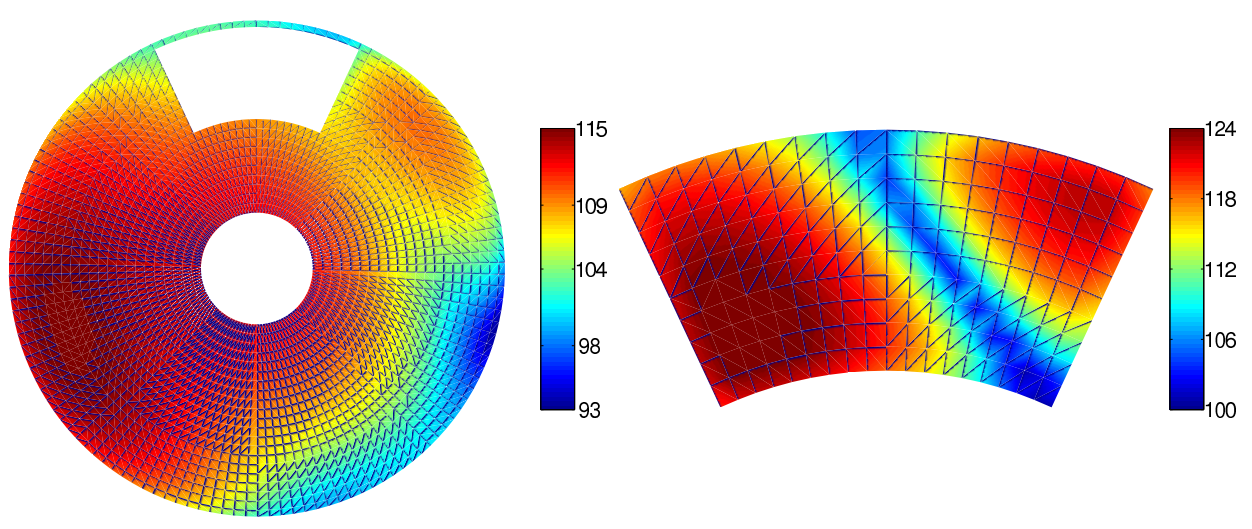

(A)

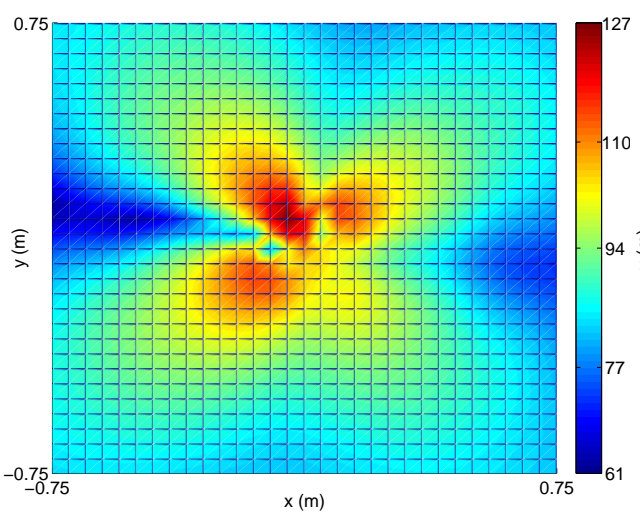

(в)

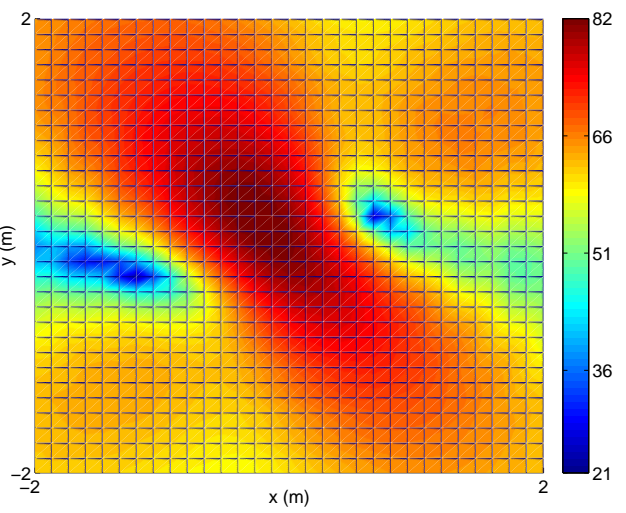

(c)

Figure 12: Acoustic intensity during stationary regime for case 1: $\mu=0.72$. (a): disc and pad (BEM); (b): near field; (c): far field

\begin{tabular}{lcc}
\hline order & harmonic component & frequency $(\mathrm{Hz})$ \\
\hline 1 & $f_{1}$ & 930 \\
& $f_{2}$ & 9423 \\
\hline 2 & $2 f_{1}$ & 1860 \\
& $-f_{1}+f_{2}$ & 8493 \\
& $f_{1}+f_{2}$ & 10.353 \\
\hline 3 & $3 f_{1}$ & 2790 \\
& $-2 f_{1}+f_{2}$ & 7563 \\
& $2 f_{1}+f_{2}$ & 11.283 \\
& $-f_{1}+2 f_{2}$ & 17.915 \\
\hline 4 & $4 f_{1}$ & 3720 \\
\hline
\end{tabular}

TABLE 5: Detected frequencies during the transient regime for case 2: $\mu=0.74$ 


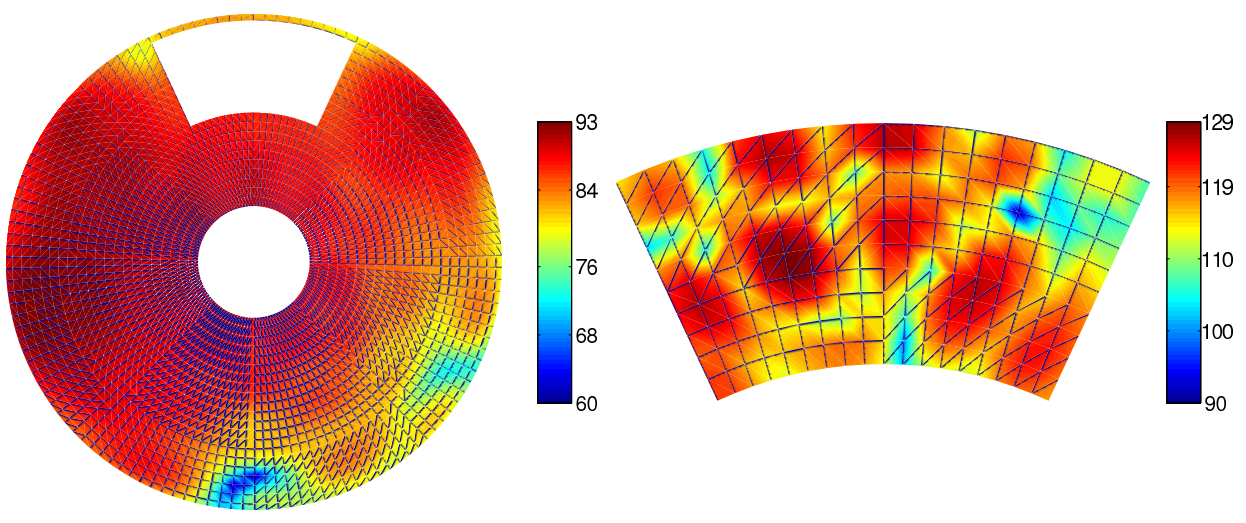

(A)

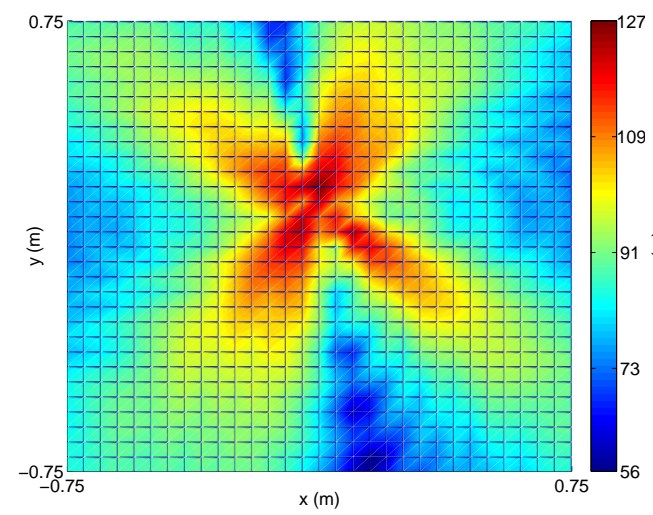

(в)

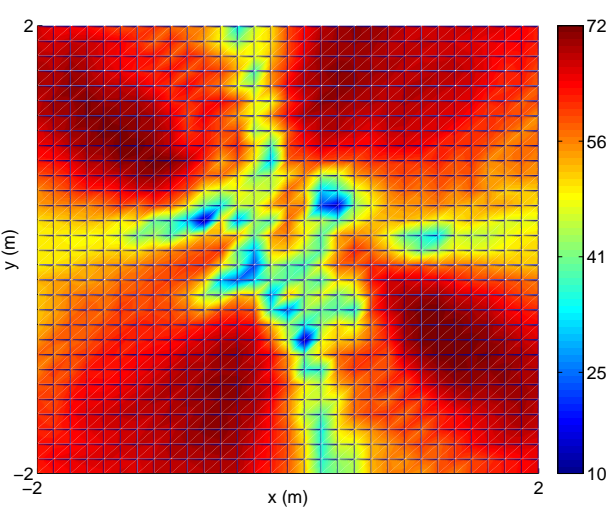

(c)

Figure 13: Acoustic intensity radiated during transient regime (zone A) for case 2: $\mu=0.74$. (a): disc and pad (BEM); (b): near field; (c): far field 
the presence of the fundamental frequencies $f_{1}$ and $f_{2}$, and also the harmonic components of the form $\pm m f_{1} \pm n f_{2}$. The sound pressure is calculated by keeping all the orders.

\begin{tabular}{lcc}
\hline order & harmonic component & frequency $(\mathrm{Hz})$ \\
\hline 1 & $f_{1}$ & 918 \\
& $f_{2}$ & 9422 \\
\hline 2 & $2 f_{1}$ & 1836 \\
& $-f_{1}+f_{2}$ & 8504 \\
& $f_{1}+f_{2}$ & 10340 \\
& $2 f_{2}$ & 18844 \\
\hline 3 & $3 f_{1}$ & 2754 \\
& $-2 f_{1}+f_{2}$ & 7586 \\
& $2 f_{1}+f_{2}$ & 11258 \\
& $-f_{1}+2 f_{2}$ & 17926 \\
& $f_{1}+2 f_{2}$ & 19762 \\
\hline 4 & $4 f_{1}$ & 3672 \\
& $-3 f_{1}+f_{2}$ & 6668 \\
& $-2 f_{1}+2 f_{2}$ & 17008 \\
\hline
\end{tabular}

TABLE 6: Detected frequencies during the final stationary regime for case 2: $\mu=0.74$

Involving case 2, Fig. 14 shows the acoustic radiation for the two unstable modes case. Fig. 14a shows the intensity $L_{d B}$ over the boundary element mesh. It can be noticed that levels change from 144 to $190 \mathrm{~dB}$ over the pad and from 81 to $141 \mathrm{~dB}$ over the disc. These levels are significantly higher than case 1 . Moreover, the propagation pattern is very different from case 1 : several local maximums of intensity are observed over the pad and the disc presents maximums of intensity along the radial direction. In near field (Fig. 14b), levels are between 181 and 250 $\mathrm{dB}$ and the pattern of directivity presents four main lobes as during zone A. It can also be noted that these four lobes have shapes and orientations that are different from those in case 1 . Involving far field (Fig. 14c), $L_{d B}$ goes from 112 to $194 \mathrm{~dB}$. As during zone A, the four previous lobes are still present but there intensities are less than in near field. For both near and far field lobes, wave frontlines are smooth due to the fact that squeal is established. Involving $P_{\theta}$, it can be seen that over $P_{0}$ and $P_{\pi / 4}$ (Fig. $15 \mathrm{~b}$ and d), the directivity pattern presents three lobes. Intensity over $P_{\pi / 2}$ presents four peculiar directions of propagation (Fig. 15f). Moreover, the acoustic intensity over $P_{3 \pi / 4}$ has several main direction of propagation but less visible than the other planes as illustrated in Fig. 15h.

Near and far field analysis show that case 2 with two unstable modes presents higher levels of noise emissions than case 1 . This can be explained by the higher amplitudes of velocity for case 2 than case 1 . In near field, directivity patterns present four lobes for both cases but there shapes and orientations are different. In far field, case 1 has only two lobes whereas case 2 presents four lobes. Finally, directivity analysis over $P_{\theta}$ shows that patterns are more complex for case 2 than case 1 . These differences are due to the different harmonic components of the velocity: for case 1 the main harmonic components are $f_{1}$ and $2 f_{1}$ whereas for case 2 , the predominant components are $f_{1}$ and $f_{2}$ and there harmonic $2 f_{1}, 3 f_{1}$ and $2 f_{2}$. 


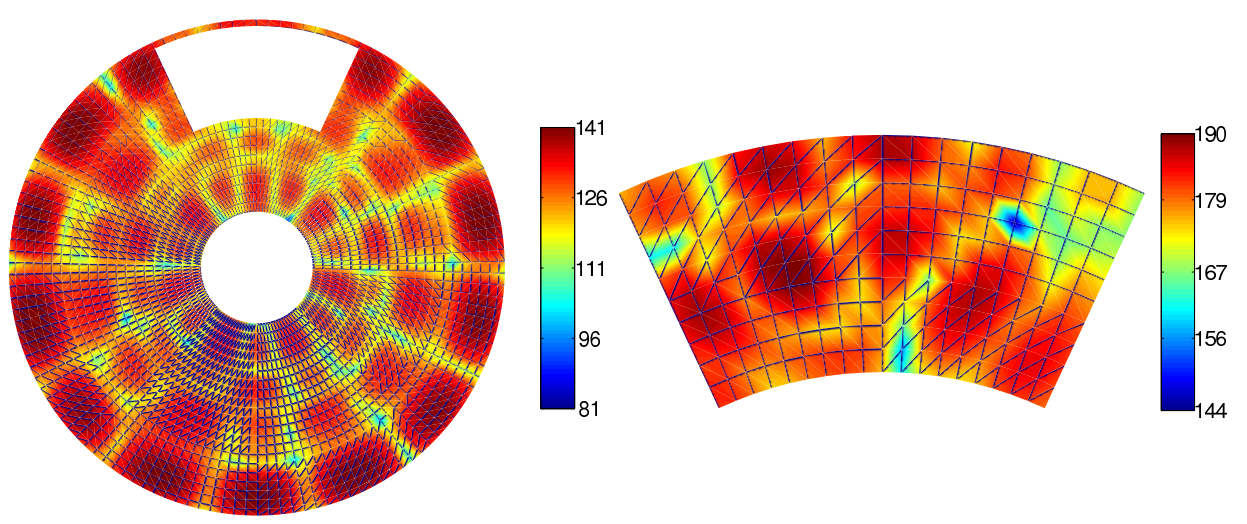

(A)

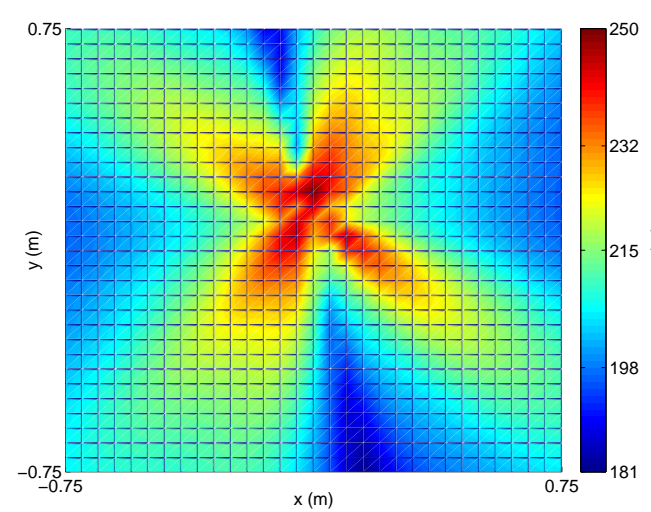

(в)

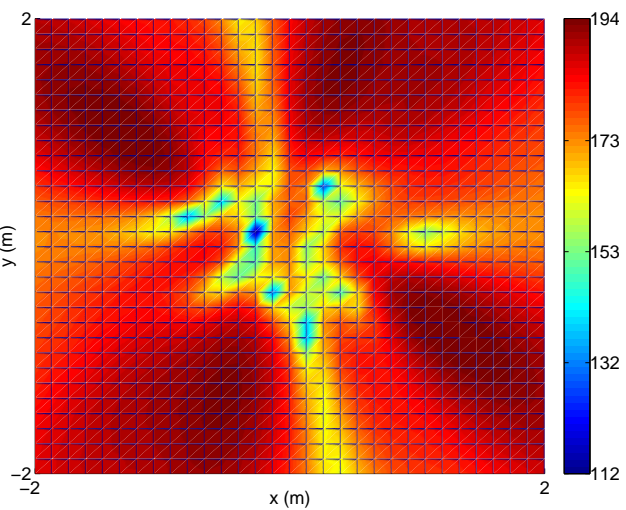

(c)

Figure 14: Acoustic intensity radiated during stationary regime (zone B) for case 2: $\mu=0.74$. (a): disc and pad (BEM); (b): near field; (c): far field 


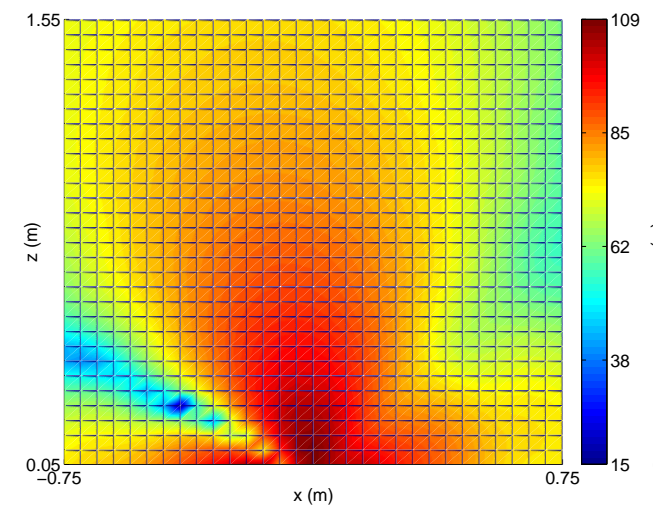

(A)

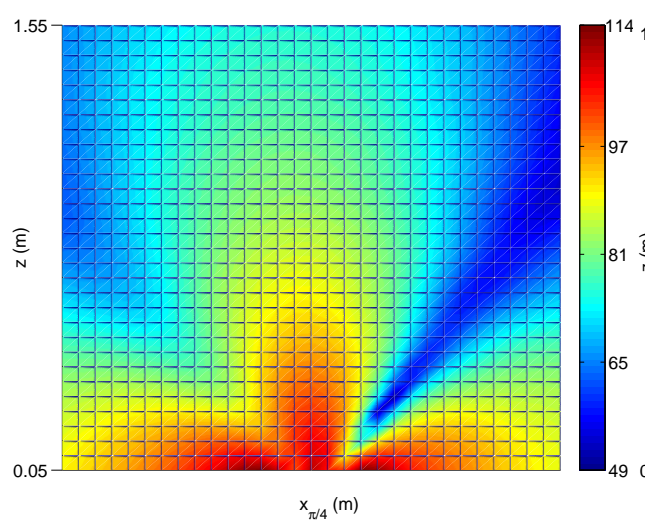

(c)

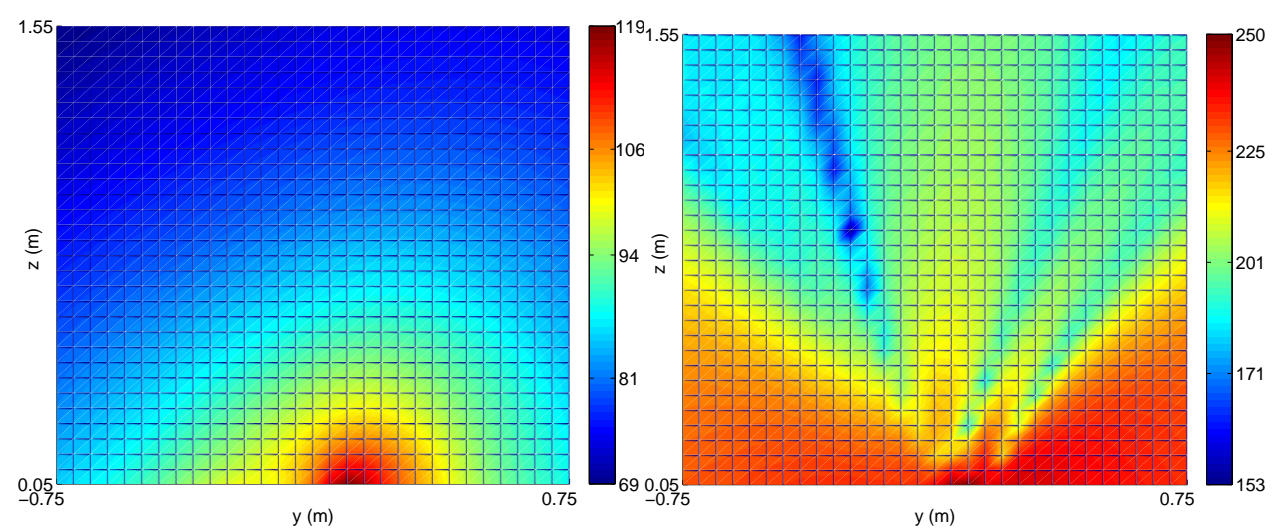

$(\mathrm{E})$

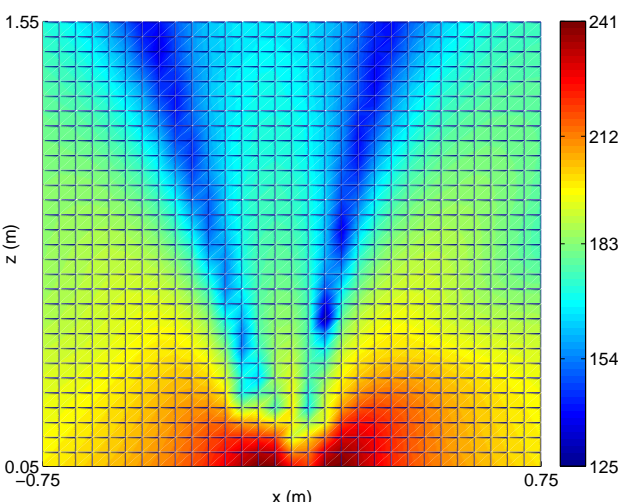

(в)

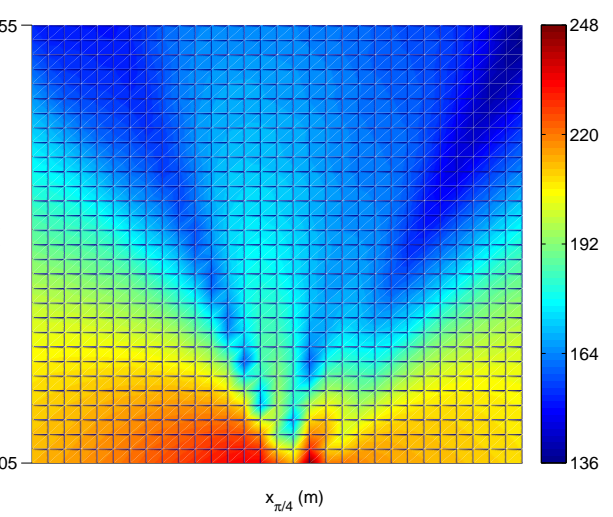

(D)

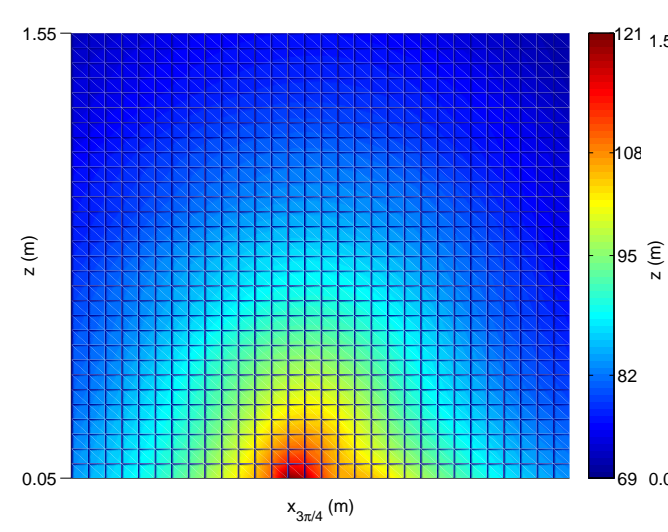

$(\mathrm{G})$

22
(F)

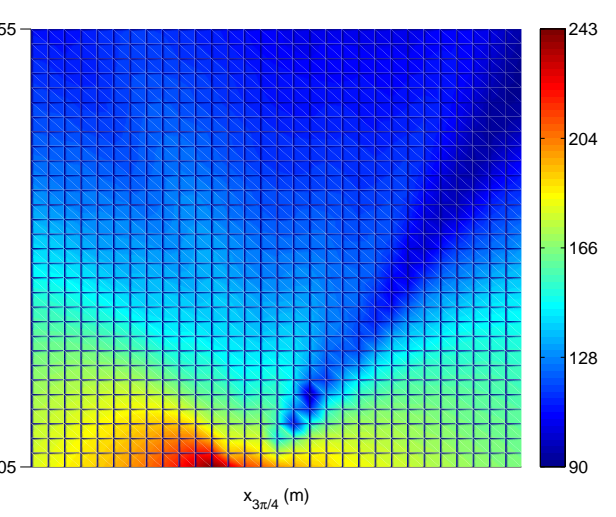

(H)

FIgURE 15: Acoustic intensity displayed over $P_{\theta}$ during stationary regmie. (a): $P_{0}$ case 1; (b): $P_{0}$ case 2 ; (c): $P_{\frac{\pi}{4}}$ case $1 ;(d): P_{\frac{\pi}{4}}$ case 2 ; (e): $P_{\frac{\pi}{2}}$ case $1 ;(f): P_{\frac{\pi}{2}}$ case $2 ;(g): P_{\frac{3 \pi}{4}}$ case 1; (h): $P_{\frac{3 \pi}{4}}$ case 2 


\subsubsection{Influence of the truncation}

The calculation of the sound pressure for the two previous cases is performed by retaining all the harmonic components (see Tables 4,5 and 6). In this section, a particular attention is payed to the influence of the truncation over the acoustic solution accuracy. We define the relative error $\epsilon_{I}$ as follow

$$
\epsilon_{I}=\frac{\left\|P_{N}-P_{I}\right\|}{\left\|P_{N}\right\|} \text { for } i=1 \ldots N
$$

where $I$ denotes the number of retained harmonic components and $N$ the total number of harmonic components. Tables 7 and 8 show the evolution of the relative error as a function of the number of retained harmonic components for case 1 and 2. Table 7 shows that the relative error is maximum when only the first component $f_{1}$ is retained. However, adding the second component $2 f_{1}$ significantly decreases the error which becomes insignificant. Then, adding more than two components does not improve the solution accuracy. That is to say, for case 1 , only the first two components lead the global sound pressure. Involving case 2, Table 8 shows that the first two components are enough to describe the global sound pressure with a small error.

\begin{tabular}{lc}
\hline retained harmonic component & relative error $\epsilon_{I}(\%)$ \\
\hline$f_{1}$ & $7.86 e^{-1}$ \\
$f_{1}, 2 f_{1}$ & $2.79 e^{-2}$ \\
$f_{1}, 2 f_{1}, 3 f_{1}$ & $1.24 e^{-2}$ \\
$f_{1}, 2 f_{1}, 3 f_{1}, 4 f_{1}$ & $1.20 e^{-2}$ \\
$f_{1}, 2 f_{1}, 3 f_{1}, 4 f_{1}, 5 f_{1}$ & $1.96 e^{-3}$ \\
$f_{1}, 2 f_{1}, 3 f_{1}, 4 f_{1}, 5 f_{1}, 6 f_{1}$ & $7.73 e^{-4}$ \\
\hline$f_{1}, 2 f_{1}, 3 f_{1}, 4 f_{1}, 4 f_{1}, 5 f_{1}, 6 f_{1}, 10 f_{1}$ & reference \\
\hline
\end{tabular}

TABLE 7: Evolution of the relative error in terms of sound pressure with respect to the number of retained harmonic components for case 1

\section{Conclusion}

First of all, this paper presents a simplified model of a brake system that is subjected to frictioninduced non-linear vibrations. It shows that the system is able to reproduce two classical cases of instabilities: single and multi-instabilities. Secondly, a method which permits to quantify noise emissions during squeal phenomenon is proposed. A boundary element model of the brake system is used in order to calculate the sound pressure radiated during squeal events. Numerical results show that levels of sound pressure increase with the friction coefficient. Moreover, it appears that the two unstable modes case present a more complex features of directivity due to the contribution of two modes than the single instability case. The truncation analysis shows that acoustic intensity is mainly led by the first two harmonic components for both cases.

\section{Aknowledgments}

The first author gratefully acknowledges the French Education Ministry which supports this research. The authors thank the financial support provided by the French National Research Agency through the framework of its project ANR-12-JS09-0009. 


\begin{tabular}{lc}
\hline retained harmonic component & relative error $\epsilon_{I}(\%)$ \\
\hline$f_{1}$ & 60.28 \\
$f_{1}, f_{2}$ & $4.35 e^{-2}$ \\
$f_{1}, f_{2}, 2 f_{1}$ & $4.36 e^{-2}$ \\
$f_{1}, f_{2}, 2 f_{1},-f_{1}+f_{2}$ & $3.67 e^{-2}$ \\
$f_{1}, f_{2}, 2 f_{1},-f_{1}+f_{2}, f_{1}+f_{2}$ & $3.44 e^{-2}$ \\
$f_{1}, f_{2}, 2 f_{1},-f_{1}+f_{2}, f_{1}+f_{2}, 2 f_{2}$ & $3.44 e^{-2}$ \\
$f_{1}, f_{2}, 2 f_{1},-f_{1}+f_{2}, f_{1}+f_{2}, 2 f_{2}, 3 f_{1}$ & $3.44 e^{-2}$ \\
$f_{1}, f_{2}, 2 f_{1},-f_{1}+f_{2}, f_{1}+f_{2}, 2 f_{2}, 3 f_{1},-2 f_{1}+f_{2}$ & $2.04 e^{-2}$ \\
$f_{1}, f_{2}, 2 f_{1},-f_{1}+f_{2}, f_{1}+f_{2}, 2 f_{2}, 3 f_{1},-2 f_{1}+f_{2}, 2 f_{1}+f_{2}$ & $1.49 e^{-2}$ \\
$f_{1}, f_{2}, 2 f_{1},-f_{1}+f_{2}, f_{1}+f_{2}, 2 f_{2}, 3 f_{1},-2 f_{1}+f_{2}, 2 f_{1}+f_{2}$, & $1.49 e^{-2}$ \\
$-f_{1}+2 f_{2}$ & \\
$f_{1}, f_{2}, 2 f_{1},-f_{1}+f_{2}, f_{1}+f_{2}, 2 f_{2}, 3 f_{1},-2 f_{1}+f_{2}, 2 f_{1}+f_{2}$, & \\
$-f_{1}+2 f_{2}, f_{1}+2 f_{2}$ & \\
$f_{1}, f_{2}, 2 f_{1},-f_{1}+f_{2}, f_{1}+f_{2}, 2 f_{2}, 3 f_{1},-2 f_{1}+f_{2}, 2 f_{1}+f_{2}$, & \\
$-f_{1}+2 f_{2}, f_{1}+2 f_{2}, 4 f_{1}$ & \\
$f_{1}, f_{2}, 2 f_{1},-f_{1}+f_{2}, f_{1}+f_{2}, 2 f_{2}, 3 f_{1},-2 f_{1}+f_{2}, 2 f_{1}+f_{2}$, & \\
$-f_{1}+2 f_{2}, f_{1}+2 f_{2}, 4 f_{1},-3 f_{1}+f_{2}$ & \\
\hline$f_{1}, f_{2}, 2 f_{1},-f_{1}+f_{2}, f_{1}+f_{2}, 2 f_{2}, 3 f_{1},-2 f_{1}+f_{2}, 2 f_{1}+f_{2}$, & \\
$-f_{1}+2 f_{2}, f_{1}+2 f_{2}, 4 f_{1},-3 f_{1}+f_{2},-2 f_{1}+2 f_{2}$ & \\
\hline
\end{tabular}

TABLE 8: Evolution of the relative error in terms of sound pressure with respect to the number of retained harmonic components for case 2 


\section{References}

[1] H. Ouyang, N. Nack, Y. Yuan, and F. Chen. Numerical analysis of automotive disc brake squeal: a review. International Journal of Vehicule Noise, 1(3-4):207-231, 2005.

[2] N. Kinkaid, O. M. O'Reilly, and P. Papadopoulos. Automotive disc brake squeal. Journal of Sound and Vibration, 267:105-166, 2004.

[3] R.A. Ibrahim. Friction-induced vibration, chatter, squeal, and chaos part 1: Mechanics of contact and friction. American Society of Mechanical Engineers Applied Mechanics Review, 47(7):209-226, 1994.

[4] R.A. Ibrahim. Friction-induced vibration, chatter, squeal, and chaos part 2: Dynamics and modeling. American Society of Mechanical Engineers Applied Mechanics Review, 47(7):227253, 1994.

[5] U. von Wagner, D. Hochlenert, and P. Hagedorn. Minimal models for disk brake squeal. Journal of Sound and Vibration, 302(3):527-539, 2007.

[6] J.-J. Sinou, F. Thouverez, and L. Jézéquel. Methods to reduce non-linear mechanical systems for instability computation. Archives of Computational Methods in Engineering, 11(3):255-342, 2004.

[7] X. Lorang, F. Foy-Margiocchi, Q.S. Nguyen, and P.E. Gautier. TGV disc brake squeal. Journal of Sound and Vibration, 293(3-5):735-746, 2006.

[8] N. Hoffmann and L. Gaul. Non-conservative beating in sliding friction affected systems: transient amplification of vibrational energy and a technique to determine optimal initial conditions. Mechanical Systems and Signal Processing, 18(3):611-623, 2004.

[9] J.-J. Sinou, G. Fritz, , and L. Jézéquel. The role of damping and definition of the robust damping factor for a self-exciting mechanism with constant friction. Journal of Vibration and Acoustics, 129(3):297-306, 2007.

[10] J.-J. Sinou. Transient non-linear dynamic analysis of automotive disc brake squeal - on the need to consider both stability and non-linear analysis. Mechanics Research Communications, 37(1):96-105, 2010.

[11] F. Massi, L. Baillet, O. Giannini, and A. Sestieri. Brake squeal: Linear and nonlinear numerical approaches. Mechanical Systems and Signal Processing, 21(6):2374-2393, 2007.

[12] A. Loyer, J.-J. Sinou, O. Chiello, and X. Lorang. Study of nonlinear behaviors and modal reductions for friction destabilized systems. application to an elastic layer. Journal of Sound and Vibration, 331(5):1011-1041, 2012.

[13] F. Chevillot, J-J. Sinou, and N. Hardouin. Nonlinear transient vibrations and coexistences of multi instabilities caused by friction in an aircraft braking system. Journal of Sound and Vibration, 328(4-5):555-574, 2009.

[14] O. Giannini, A. Akay, and F. Massi. Experimental analysis of brake squeal noise on a laboratory brake setup. Journal of Sound and Vibration, 292(1-2):1-20, 2006.

[15] F. Chevillot, J.-J. Sinou, and N. Hardouin. Simulations and experiments of a nonlinear aircraft braking system with physical dispersion. Journal of vibration and acoustics, 132(4):041010, 11 pages, 2010. 
[16] T. Butlin and J. Woodhouse. A systematic experimental study of squeal initiation. Journal of Sound and Vibration, 330(21):5077-5095, 2011.

[17] N. Coudeyras, S. Nacivet, and J.-J. Sinou. Periodic and quasi-periodic solutions for multiinstabilities involved in brake squeal. Journal of Sound and Vibration, 328(4-5):520-540, 2009 .

[18] G. Fritz, J.-J. Sinou, J.-M. Duffal, and L. Jézéquel. Effects of damping on brake squeal coalescence patterns - application on a finite element model. Mechanics Research Communications, 34(2):181-190, 2007.

[19] Marc Bonnet. Boundary Integral Equation Methods for Solids and Fluids. John Wiley \& Sons Ltd, April 1999.

[20] V. Cutanda Henriquez and P. Juhl. OpenBEM. http://www.openbem.dk/. 